\title{
Effects of Different Electroacupuncture Scheduling Regimens on Murine Bone Tumor-Induced Hyperalgesia: Sex Differences and Role of Inflammation
}

\author{
Branden A. Smeester, ${ }^{1}$ Mona Al-Gizawiy, ${ }^{2}$ and Alvin J. Beitz ${ }^{1,3}$ \\ ${ }^{1}$ Department of Veterinary and Biomedical Sciences, College of Veterinary Medicine, University of Minnesota, St. Paul, \\ MN 55108, USA \\ ${ }^{2}$ Department of Radiology, Medical College of Wisconsin, Milwaukee, WI 53226, USA \\ ${ }^{3}$ Department of Veterinary and Biomedical Sciences, University of Minnesota, Room 205 Veterinary Medicine Building, \\ 1971 Commonwealth Avenue, St. Paul, MN 55108, USA
}

Correspondence should be addressed to Alvin J. Beitz, beitz001@umn.edu

Received 23 August 2012; Revised 21 November 2012; Accepted 28 November 2012

Academic Editor: Chong-Zhi Wang

Copyright (C) 2012 Branden A. Smeester et al. This is an open access article distributed under the Creative Commons Attribution License, which permits unrestricted use, distribution, and reproduction in any medium, provided the original work is properly cited.

\begin{abstract}
Previous studies have shown that electroacupuncture (EA) is able to reduce hyperalgesia in rodent models of persistent pain, but very little is known about the analgesic effects and potential sex differences of different EA treatment regimens. In the present study, we examined the effects of five different EA treatments on tumor-induced hyperalgesia in male and female mice. EA applied to the ST-36 acupoint either twice weekly (EA-2X/3) beginning on postimplantation day (PID) 3 or prophylactically three times prior to implantation produced the most robust and longest lasting antinociceptive effects. EA treatment given once per week beginning at PID 7 only produced an antinociceptive effect in female animals. The analgesic effect of EA-2X/3 began earlier in males, but lasted longer in females indicating sex differences in EA. We further demonstrate that EA-2X/3 elicits a marked decrease in tumor-associated inflammation as evidenced by a significant reduction in tumor-associated neutrophils at PID 7. Moreover, EA-2X/3 produced a significant reduction in tumor-associated $\mathrm{PGE}_{2}$ as measured in microperfusate samples. Collectively, these data provide evidence that EA-2X/3 treatment reduces tumor-induced hyperalgesia, which is associated with a decrease in tumorassociated inflammation and $\mathrm{PGE}_{2}$ concentration at the tumor site suggesting possible mechanisms by which EA reduces tumor nociception.
\end{abstract}

\section{Introduction}

Although acupuncture remains a controversial subject in Western Medicine, it is widely used in today's clinical practice [1] and appears to be a viable option for the treatment of many types of pain [2-4] including cancer pain $[5,6]$ and chemotherapy-induced peripheral neuropathy $[7,8]$. Cancer is a highly destructive and infiltrative disease resulting in uncontrolled proliferation, apoptotic deregulation, and invasive metastasis. Up to $90 \%$ of patients with metastatic or advanced stage cancer will experience significant cancerrelated pain. Bone metastases are often associated with dramatic reductions in quality of life, mobility, and independence as well as excruciating refractory pain [9]. In a recent pan-European survey, 63\% of patients on prescribed analgesics experienced breakthrough cancer pain [10]. While some progress had been made in attempting to elucidate the mechanisms responsible for tumor-induced bone pain [11-13], the potential role of inflammation in bone tumor pain and the mechanisms underlying breakthrough pain are poorly understood. Furthermore, although opiates are a mainstay of cancer pain treatment, recent work has shown that morphine accelerates sarcoma-induced bone pain, bone loss, and spontaneous fractures [14]. In this regard, it is critical that further research be done to better understand bone cancer mechanisms, how inflammation affects bone cancer progression, and if alternative treatments can be found that both relieve pain and reduce tumor burden. 
While acupuncture has been around for thousands of years, we are only now starting to get a glimpse of the mechanisms underlying its effects [15] particularly those associated with pain relief $[16,17]$. As we begin to study the mechanisms underlying acupuncture's analgesic effects, there are important questions that arise regarding whether different acupuncture treatment regimens have different effects on pain and whether sex differences exist related to these treatment regimens. In the present study we hypothesized that different treatment regimens would have differential antinociceptive effects on tumor-induced pain. We also wanted to determine if there were sex differences among different acupuncture treatment schedules, since this is an area that has received little attention in the literature. Thus, the first portion of the present study was designed to examine the effectiveness of five different EA treatment regimens in reducing bone tumor pain and to also determine whether these regimens had differential effects in male versus female animals.

Acupuncture also appears to affect the immune system and therefore can have a direct effect on inflammation $[18,19]$, including reducing neutrophil invasion during sepsis [20]. The role of inflammation in carcinogenesis has been extensively investigated and well documented [21-23]. Neurogenic inflammation appears to underlie certain forms of colon cancer [24]. In this regard, the inflammatory state is necessary to maintain and promote cancer progression and to accomplish the full malignant phenotype, including tumor tissue remodeling, angiogenesis, metastasis, and the suppression of the innate anticancer immune response [25]. While several studies now support acupuncture's ability to reduce inflammation $[26,27]$, its potential effect on the inflammatory stage of cancer or the inflammatory component of cancer pain has not been examined. In the present study, we hypothesized that low-frequency electroacupuncture (EA) has an anti-inflammatory effect on neutrophil and macrophage recruitment during early tumor development and that acupuncture-induced inhibition of tumor-associated inflammation reduces tumorinduced hyperalgesia. To test these hypotheses, we utilized an established hind paw osteosarcoma mouse model developed at the University of Minnesota $[28,29]$ in the second part of this study to examine the above relationships in vivo.

\section{Materials and Methods}

2.1. Cells. The K7 M2 cell line was a kind gift from Dr. Khanna [30] and was derived from the K7 cell lines initially established from a spontaneous murine OGS [31]. National Collection of Type Cultures (NCTC) clone 2472 fibrosarcoma cells, originally derived from a connective tissue tumor in a $\mathrm{C} 3 \mathrm{H}$ mouse, were obtained from the American Type cell Culture Collection (Rockville, MD). Each cell type is syngeneic to its particular mouse line. Cells were grown and maintained in accordance with standard cell culturing techniques. K7 M2 cells were grown to $80 \%-$ $90 \%$ confluence in $75 \mathrm{~cm}^{2}$ flasks (Corning, Lowell, MA) in Dulbecco's Modification of Eagles Medium (Invitrogen,
Carlsbad, CA) containing 1\% penicillin/streptomycin and $10 \%$ fetal bovine serum prior to implantation. NCTC 2472 cells were grown using a similar protocol, fortified with $10 \%$ horse serum, sodium bicarbonate, and also grown to $80 \%-90 \%$ confluency. Cell cultures were housed in a waterjacketed incubator with $5 \%$ carbon dioxide at $37^{\circ} \mathrm{C}$.

2.2. Animals. The present study used a well-established mouse hind paw model of bone cancer pain $[13,28]$ to examine the effects of EA on tumor-induced nociception. A total of 104 female and 166 male young adult BALB/c and 20 male $\mathrm{C} 3 \mathrm{H}$ mice (National Cancer Institute, Bethesda, MD) were used in this study. The number of animals in each group that were used for each experiment is summarized in Table 1.

The inbred BALB/c mouse strain was used in these experiments because this mouse strain is syngeneic to the K7 M2 osteosarcoma used in these experiments and thus allows these cells to grow tumors without rejection. Osteosarcoma tumors were studied in the present investigation because osteosarcoma is an extremely painful bone tumor that affects children and teenagers and an equally painful tumor in dogs, and we wanted to investigate the potential for EA to treat osteosarcoma-induced bone pain; $\mathrm{C} 3 \mathrm{H} / \mathrm{He}$ mice were used for the microperfusion experiments in the present study because they are syngeneic to the fibrosarcoma cells used for these experiments. Thus, BALB/c mice were used for behavioral and immunohistochemical experiments, while $\mathrm{C} 3 \mathrm{H}$ mice were used for microperfusion experiments. The $\mathrm{C} 3 \mathrm{H}$ mice were selected for the microperfusion experiments, because based on preliminary data we are able to obtain more robust and reproducible results when performing microperfusion experiments on fibrosarcoma tumors as compared to osteosarcoma tumors in vivo. Animals were maintained on a $12 \mathrm{hr}$ light/dark cycle with food and water ad libitum. Procedures were performed in accordance with the guidelines recommended by the International Association for the Study of Pain, and all experimental protocols were approved by the Animal Care and Use Committee at the University of Minnesota.

2.3. Implantation. Cells were prepared for implantation by first pouring off the culture medium and washing with PBS. Trypsin was added to flask and placed in incubator for $3 \mathrm{~min}$. Upon detachment, cells were suspended in an ample amount of culture medium to terminate enzymatic activity and centrifuged for $10 \mathrm{~min}$ at 1,000 g. Osteosarcoma and fibrosarcoma cells were resuspended in a known amount of PBS for counting, quantified on a hemocytometer, pelleted and re-suspended in PBS. Osteosarcoma cells were resuspended to a final concentration of $1 \times 10^{6}$ cells in $50 \mu \mathrm{L}$, while fibrosarcoma cells were re-suspended to a final concentration of $2 \times 10^{5}$ cells in $10 \mu \mathrm{L}$. Initially, animals were anesthetized in a plexi-glass chamber using $3 \%$ isoflurane in $3 \mathrm{~L} / \mathrm{min}$ oxygen. Once each mouse was completely anesthetized, a maintenance rate of $2 \%$ isoflurane in $1.5 \mathrm{~L} / \mathrm{min}$ oxygen was maintained during the short implantation procedure. Tumor cells were manually injected by boring into the calcaneus bone using a 29.5 gauge needle connected to a sterile $0.3 \mathrm{~mL}$ 
TABLE 1: Summary of the total number of male and female animals used for each experiment.

\begin{tabular}{|c|c|c|c|}
\hline Assay & $\begin{array}{c}\text { Treatment } \\
\text { type }\end{array}$ & $\begin{array}{l}\text { Male } \\
(n)\end{array}$ & $\begin{array}{c}\text { Female } \\
(n)\end{array}$ \\
\hline \multirow{4}{*}{$\begin{array}{l}\text { Mechanical } \\
\text { Hyperalgesia von Frey }\end{array}$} & Tumor & 24 & 15 \\
\hline & Saline & 11 & 7 \\
\hline & EA & $\begin{array}{c}52 \\
\text { (10-11/group) }\end{array}$ & $\begin{array}{c}45 \\
\text { (9/group) }\end{array}$ \\
\hline & Sham & $\begin{array}{c}30 \\
\text { (6/group) }\end{array}$ & $\begin{array}{c}37 \\
\text { (7-8/group) } \\
\end{array}$ \\
\hline \multirow{4}{*}{ Immunohistochemistry } & Tumor & 10 & 0 \\
\hline & EA & 10 & 0 \\
\hline & Sham & 10 & 0 \\
\hline & N/A & 10 & 0 \\
\hline \multirow{5}{*}{ Microperfusion/ELISA } & Tumor & 3 & 0 \\
\hline & Saline & 3 & 0 \\
\hline & $\mathrm{Sal}+\mathrm{EA}$ & 3 & 0 \\
\hline & Tum + EA & 3 & 0 \\
\hline & & 186 & 104 \\
\hline
\end{tabular}

insulin syringe as previously described [28]. A control group of BALB/c mice $(n=10)$ received an injection of saline into the calcaneus bone rather than tumor cells. These mice were used for the initial behavioral testing. Following injection, mice were allowed to recover in cages on a heating pad. Animals showing any signs of dysfunction (e.g., problems with ambulation, lethargy, or excessive bleeding) or any animals in which the tumor did not grow were euthanized and removed from the study. This occurred in less than $5 \%$ of the animals used in this study.

2.4. Behavioral Testing. Animals were placed under clear glass cups on a wire grid and allowed to acclimate for 30 min. Mechanical hypersensitivity was tested using a von Frey filament \# 2.83, which produces a force of $62-$ $95 \mathrm{mg}$ [32], and was applied to the plantar surface of each hind paw with enough force to cause it to bow slightly. Starting with the right hind paw, the numbers of positive responses out of a total of 10 applications were recorded. Baseline von Frey measurements were obtained prior to tumor implantation or saline injection into the calcaneus. Subsequent von Frey measurements were taken 30 min after each electroacupuncture treatment as well as on days 3, 7, $10,14,17$, and 21. Animals with saline injection into the calcaneus did not receive any EA treatment, but rather served as controls for tumor cell implantation and tumor-induced nociception. Behavioral assessments were conducted during the light cycle at approximately the same time each day. The investigator performing the von Frey testing was blinded to the animal and EA treatment (Tumor-EA versus TumorSham EA versus Tumor-non-acupoint EA versus No TumorNo EA, i.e., saline implantation).

2.5. Transcardiac Perfusions. Mice were deeply anesthetized with $50 \mathrm{mg} / \mathrm{kg}$ sodium pentobarbital (Nembutal; Ovation
Pharmaceuticals, Inc., Deerfield, IL) injected intraperitoneally. When mice were no longer responsive to paw pinch, the thoracic cavity was quickly accessed via the abdomen to isolate the heart. A 21-gauge butterfly catheter (Terumo Medical Corporation, Somerset, NJ) was inserted into the left ventricle and secured using forceps. The right atrium was punctured to allow both drainage of blood and fixative. Fifteen $\mathrm{mL}$ of ice cold PBS was perfused followed by $30 \mathrm{~mL}$ of $10 \%$ neutral-buffered formalin at a rate of $3 \mathrm{~mL} / \mathrm{min}$. Following perfusion, tumors were excised, postfixed in the same fixative overnight at $4^{\circ} \mathrm{C}$, and cryoprotected in $30 \%$ sucrose for $24-48$ hours at $4^{\circ} \mathrm{C}$ prior to tissue sectioning.

2.6. Microperfusions. Microperfusion of the tumor was performed as previously described [28] in order to examine the potential effect of EA on the concentration of prostaglandin $\mathrm{E}_{2}$ in the extracellular fluid of a hind paw fibrosarcoma. Previous microperfusion studies in our lab have been conducted on $\mathrm{C} 3 \mathrm{H}$ mice, and, thus, we have established baseline levels for prostaglandins, cytokines, and other potential pain mediators in this mouse line. Moreover, we have found in preliminary experiments that the microperfusion technique has a higher success rate in $\mathrm{C} 3 \mathrm{H}$ mice with fibrosarcoma tumors then in BALB/c mice with osteosarcomas. Since hind paw fibrosarcoma tumors in $\mathrm{C} 3 \mathrm{H}$ mice produce a level of mechanical hyperalgesia that is similar to that of osteosarcoma tumors [28] and since EA also produces antinociception in this mouse strain, we evaluated the effect of EA on the concentration of prostaglandin $E_{2}$ in fibrosarcoma tumors rather than osteosarcomas. Briefly, we implanted a perfusion microprobe consisting of a 29.5-gauge stainless steel hypodermic needle with a $2 \mathrm{~mm}$ opening in the middle, extending a "push-pull" microperfusion design as previously described [28] into the tumor site, and secured the probe with super glue. Using two peristaltic pumps (Rabbit Plus, Rainin Instrument $\mathrm{Co}$, Columbus, $\mathrm{OH}$ ), we utilized this push-pull technique to collect tumor secretions at various time points following EA or sham treatments. The tumor was perfused with a modified Ringer's Solution at a flow rate of $10 \mu \mathrm{l} / \mathrm{min}$. Preliminary experiments have determined that this setting on the outlet pump maintained a constant pressure at the microprobe opening and prevented clogging of the probe. Heparin $(2 \mu \mathrm{l})$ was used to prevent potential blood clotting in the microprobe. Polyethylene tubing (PE-10) was used to connect the peristaltic pumps with the microprobe. Animals were initially anesthetized in a plexi-glass chamber using $3 \%$ isoflurane in $3 \mathrm{~L} / \mathrm{min}$ oxygen. Once animals were anesthetized as measured by paw pinch, a maintenance rate of $2 \%$ isoflurane in $1.5 \mathrm{~L} / \mathrm{min}$ oxygen was used during the microperfusion procedure. After a $30 \mathrm{~min}$ equilibration period, fractions were collected in 30-minute increments before treatment $(\mathrm{BL})$ and at two time points following EA or sham treatment. Samples were collected and augmented with a protease inhibitor mixture (Sigma, St. Louis, MO). They were then stored at $-80^{\circ} \mathrm{C}$ for later analysis by ELISA assay.

2.7. EA Stimulation Parameters. For EA, sham, or nonacupoint treatment, animals were initially anesthetized with 
$3 \%$ isoflurane $/ 3 \mathrm{~L}$ oxygen and then maintained at $2 \%$ isoflurane/1.5 L oxygen for the length of the EA, sham, or nonacupoint procedure $(30 \mathrm{~min})$. For the EA and sham-EA acupuncture groups, two stainless steel intradermal needles (SEIRIN-America, Weymouth, MA) were inserted to a depth of $3 \mathrm{~mm}$ into the hind limb at the ST-36 Zusanli acupoint located between the tibia and fibula, approximately $5 \mathrm{~mm}$ lateral to the anterior tubercle of the tibia. Rather than testing multiple acupoints and examining their effects on tumor nociception, we focused on one point, Zusanli, for the purposes of this study. This allowed us to evaluate differences in the frequency and timing of the EA application, rather than the effect of stimulating different individual acupoints or simultaneous stimulation of multiple acupoints. The Zusanli acupuncture point was selected because it has been used to evaluate the effect of EA on a variety of pain conditions in rodent models of persistent and chronic pain and has been found to produce significant analgesia $[33,34]$. In the EA groups, the Zusanli acupoint was stimulated using a Maxtens 1000 dual channel stimulator with a $4 \mathrm{~Hz}$ pulse rate, $100 \mu \mathrm{s}$ pulse width, for a total of $30 \mathrm{~min}$. A similar paradigm was used to stimulate a nonacupoint in Group four (see the following).

2.7.1. Electroacupuncture Treatment Regimens. To our knowledge, there are very few controlled studies in the literature that have examined the effects of different acupuncture regimens (treatment parameters) on pain. The present study examines and compares the potential antinociceptive effects of five different EA treatment paradigms administered at the ST-36 acupoint in a rodent model of bone cancer pain. Three of our regimens, EA given twice per week and EA given once per week, are based on those used clinically to treat pain $[5,35]$. The five treatment protocols were as follows: EA administered once weekly starting at PID 7 (EA-1X/7); EA administered twice weekly starting at PID $3(\mathrm{EA}-2 \mathrm{X} / 3)$ or PID $5(\mathrm{EA}-2 \mathrm{X} / 5)$; EA administered once on day 1 following tumor cell implantation (EA Once/1); and EA administered 3 times prior to tumor cell implantation with no treatment following implantation, which we designated as the prophylactic (EA Pro) treatment subgroup. Male and female osteosarcoma tumor-bearing animals were divided into the following four experimental and control groups: Group 1: an EA treated tumor group, which consisted of 5 subgroups of animals all implanted with tumor cells but each receiving a different EA treatment regimen as described previously (EA-1X/7, EA-2X/3, EA2X/5, EA Once/1, or EA Pro); Group 2: a sham-EA treated tumor group, which consisted of 5 subgroups of animals all implanted with tumor cells, but each receiving a different sham-EA treatment regimen (similar to the EA treatment regimens described previously) that involved implanting a acupuncture needle into ST-36, but not applying any electrical current (Sham-1X, Sham-2X/3, Sham-2X/5, Sham Once/1, and Sham Pro); Group 3: a Tumor-No EA treatment group (mice that were implanted with tumor cells, but received no EA or sham treatment and were subsequently euthanized at day 21 after implantation; $n=10$ ); and
Group 4: a nonacupoint EA treated tumor group (N/A-2X/3; $n=10$ ), which received EA twice a week in a nonacupoint located on the base of the tail as previously described by Kim et al. [36]. Similar to group one, the non-acupoint treatment group received twice weekly EA at the non-acupoint site for a period of 21 days.

Sham treatment consisted of acupuncture needles being inserted into the ST-36 acupoint for $30 \mathrm{~min}$, without application of electrical stimulation. Previous studies indicate no significant differences in nonpenetrating sham needles, hence our omission of this control. [37-39]. However, in accordance with the reasoning that acupuncture analgesia is dependent on the mechanical signal elicited by needle manipulation, our sham EA group had the needles inserted, but there was no EA current or mechanical stimulation [4044].

2.8. Immunohistochemistry for Immunocytes. Because inflammation is a component of many tumor types and since acupuncture has been shown to reduce inflammation, we hypothesized that acupuncture may reduce tumor-induced nociception by reducing tumor-associated inflammation. Here we use immunocytochemistry to analyze the potential effects of EA on neutrophils and macrophages at the tumor site. Since the EA-2X/3 treatment schedule provided the most effective and long lasting antinociceptive effect, this treatment schedule was selected to examine the potential effects of EA on tumor-associated inflammation. Osteosarcoma tumor-bearing hind limbs were removed immediately after transcardiac perfusion, postfixed in $10 \%$ neutralbuffered formalin overnight at $4^{\circ} \mathrm{C}$, and then placed in $30 \%$ sucrose for cryoprotection. Consecutive tissue sections (40 microns) through the distal third $(2.5 \mathrm{~mm})$, the middle third $(2.5 \mathrm{~mm})$, and the proximal third $(2.5 \mathrm{~mm})$ of the tumor were cut transversely using a cryostat. Tissue sections through each third of the tumor were separated by $300 \mu \mathrm{m}$ increments to avoid double counting of cell numbers. This allowed us to sample the entire tumor (average tumor diameter was $7.5 \mathrm{~mm}$ in diameter). Thus, a total of 30 sections ( 10 from the distal third, 10 from the middle third, and 10 from the proximal third) through the tumor of each mouse were collected and processed using a standard immunofluorescent procedure. Six tumor sections were randomly selected from each third of the tumor and were blocked for $1 \mathrm{hr}$ with $0.3 \%$ Triton X-100/PBS (TPBS) and $5 \%$ donkey serum at RT. Three of the randomly selected tissue sections from each third of the tumor ( 9 sections total) were then incubated overnight at $4^{\circ} \mathrm{C}$ with rat anti-mouse NIMP-R14 (1:50; Abcam, Cambridge, MA) to specifically label neutrophils. The remaining nine randomly selected tissue sections from the tumor were incubated overnight with rat anti-mouse MOMA-2 (1:50; Abcam, Cambridge, MA) to specifically label macrophages/monocytes at the tumor site. Following $3 \times 10 \mathrm{~min}$ PBS washes, sections were incubated for $2 \mathrm{hr}$ at RT using Cy 3-conjugated AffiniPure Donkey Anti-Rat IgG (1 : 400; Jackson Immuno, West Grove, $\mathrm{PA})$. Sections were washed $3 \times 10 \mathrm{~min}$ in PBS, air-dried for $15 \mathrm{~min}$, and mounted using Pro-Long Gold mounting 
medium containing 4',6-diamidino-2-phenylindole (Invitrogen, Carlsbad, CA). In addition, 2 tissue sections from each third of the tumor were used as controls and were processed using immunocytochemistry with either the primary or secondary antibody left out of the process to determine possible nonspecific staining.

2.9. Immunocyte Quantitative Analysis. The quantification of neutrophils and macrophages in tumor sections was performed based on the computer-assisted cell counting method recently developed by Väyrynen and coworkers [45]. Peripheral osteosarcoma tumor sections from the left hind paw were analyzed at 40X magnification using a Nikon Eclipse 80i (Nikon, Melville, NY) microscope to determine the densities of neutrophils and macrophages. Tissue sections were thresholded using preset software parameters in order to insure consistency among fields of view and slides per animal. Three random fields were selected from the peripheral portion of the tumor in each section, and the density of cells in each field was determined. A total of 7 sections per antibody were analyzed per animal. In addition, 6 control sections were analyzed from each animal in which the primary or secondary antibody was omitted from the immunohistochemical processing. For these controls, the tissue was incubated in blocking solution without the primary antibody or with PBS rather than the secondary antibody. These sections served as controls for nonspecific staining. Since we did not have access to the original antigens used to generate the antibodies used in this study, we were unable to perform appropriate adsorption controls. Nikon ACT-1 (Nikon, Melville, NY) software was used to acquire the images for processing. Individual cell types (neutrophils or macrophages) were identified by a brighter immunofluorescent staining pattern visible against the darker background. In addition, we used a DAPI counterstain and $\mathrm{H} \& \mathrm{E}$ staining to positively identify cell types. Neutrophils were characterized by their multilobed nucleus and pink cytoplasm, while macrophages are larger cells with pleomorphic nuclei and pale gray-blue cytoplasm that often contain vacuoles. Image software (National Institutes of Health, Bethesda, MA) was utilized for pixel density collection and analysis. The quantitative analysis that was performed on tumor sections was done entirely by an investigator blinded to the experimental conditions.

\subsection{Enzyme-Linked Immunosorbent Assay (ELISA) Mea-} surement of Prostaglandin $E_{2}$ Supernatant. A serological testfor prostaglandin $\mathrm{E}_{2}$ was performed using a commercially available EIA kit (Caymen Chemical Company, Ann Arbor, $\mathrm{MI})$. An anti- $\mathrm{E}_{2}$ monoclonal antibody was used on microperfusate samples collected from the hind paw fibrosarcoma tumor 30 minutes prior to the EA procedure (to determine baseline concentrations) as well as three consecutive 30minute fractions during and after treatment. Samples were run in duplicate, read at $540 \mathrm{~nm}$ on a photospectrometer, and analyzed using Caymen Chemical company software. This analysis was performed by an investigator blinded to the experimental condition.
2.11. Statistical Analysis. Complete statistical analyses of all data sets were carried out. Comparisons between groups were performed using a two-way ANOVA with post hoc comparisons using Bonferroni's method. For single time point comparisons between groups or within a group, an unpaired Student's $t$-test was employed. Wherever necessary, a paired Student's $t$-test was used to test for statistical significance at individual time points. Data was analyzed and graphed using Prism 5.0 (GraphPad Software, La Jolla, CA). The level of significance was set at $P \leq 0.05$.

\section{Results}

3.1. Tumor-Induced Hyperalgesic Behavior. Implantation of osteosarcoma cells into the hind paw of $\mathrm{BALB} / \mathrm{c}$ mice induced hyperalgesia to normally nonnoxious mechanical stimuli as measured using a von Frey monofilament (2.83) when compared to their saline injected controls (Figure 1(a)); ${ }^{*} P<0.05,{ }^{*} P<0.01,{ }^{*} * P<0.001$, $* * * * P<0.0001$; C3H fibrosarcoma-induced hyperalgesia data is shown in Figure 1(b) and is similar to that published previously [28]. This tumor-induced mechanical hyperalgesia, as defined by a significant increase in the number of responses to von Frey monofilament application, was evident as early as postimplantation day 3 (PID) and continued throughout the duration of the experiment.

3.2. Osteosarcoma versus Fibrosarcoma Hyperalgesia Control. Implantation of either the K7M2 cell line or the NCTC 2472 cell line produced significant tumor-induced mechanical hyperalgesia through PID 14 (Figure 1(b)). There was no significant difference in the amount of hyperalgesia between the two cell lines (Figure 1(b); $P>0.05$ ). EA-2X/3 produced a significant decrease in tumor-induced hyperalgesia in both fibrosarcoma and osteosarcoma implanted mice.

\subsection{Effect of Different Treatment Regimens: EA Treatment at ST-36 Significantly Attenuates Tumor-Induced Hyperalge- sia}

3.3.1. EA-1X/7 (EA Administered Once per Week Beginning at $P I D$ 7). EA-1X/7 produced a significant decrease in tumorinduced mechanical hyperalgesia in both male and female animals that was evident at $15 \mathrm{~min}$ after EA stimulation, peaked at $30 \mathrm{~min}$ post-treatment and returned to pre-EA levels by $60 \mathrm{~min}$ after treatment (data not shown). This immediate antinociceptive effect of EA was also evident in the EA-2X/3, EA-2X/5, and EA-once/1 treated animals, but not in any of the sham-EA treated animals. However, when the effects of treatment over time were evaluated, EA-1X/7 (Figure 1(b)) significantly attenuated mechanical hyperalgesia only on PID 7 in female cancer-bearing animals when compared to the Tumor-No EA control group $(* * P<$ $0.01)$. Conversely EA treatment given once a week had no effect on tumor-induced hyperalgesia in male tumor mice at any of the time points tested. Sham treatment given 


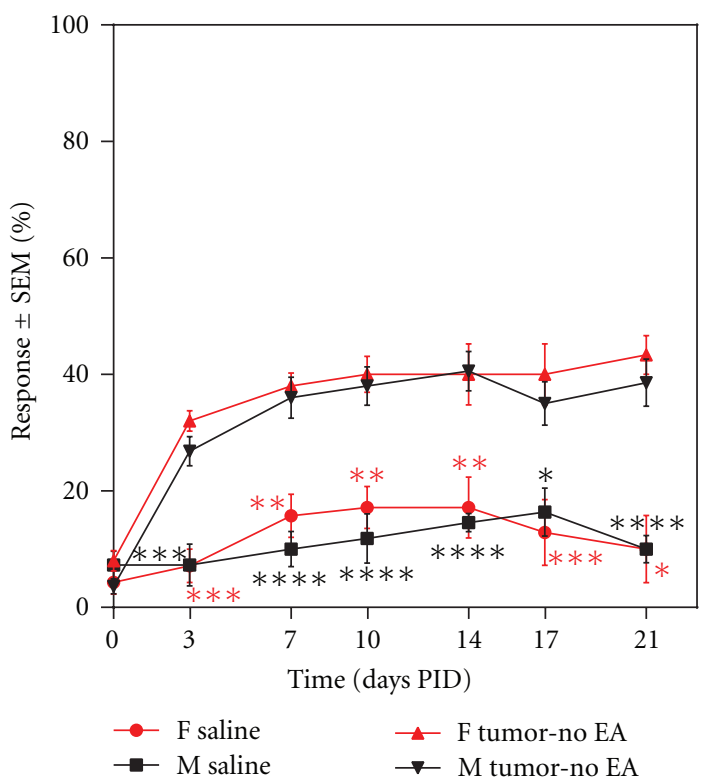

(a) MF Saline versus MF Tumor

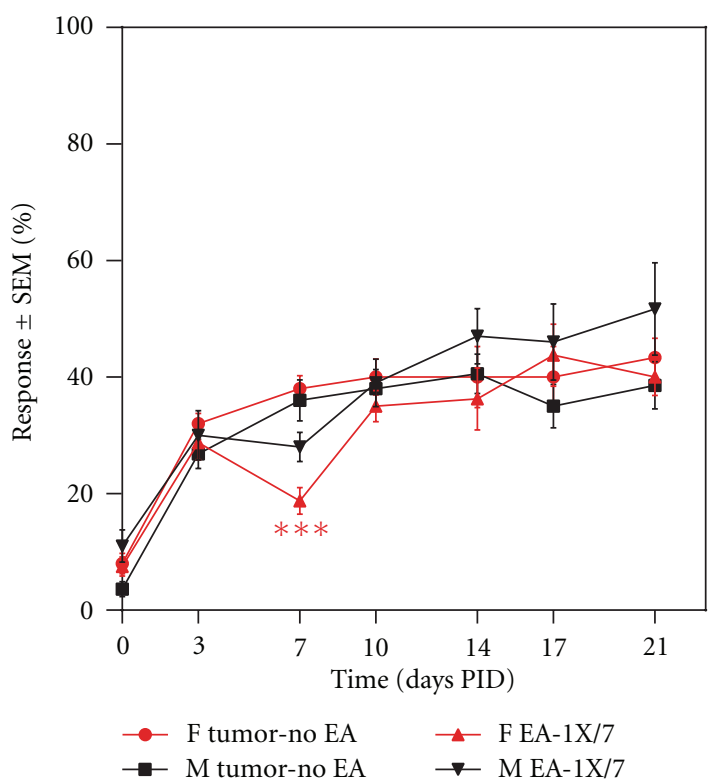

(c) MF Tumor versus MF EA-1X/7

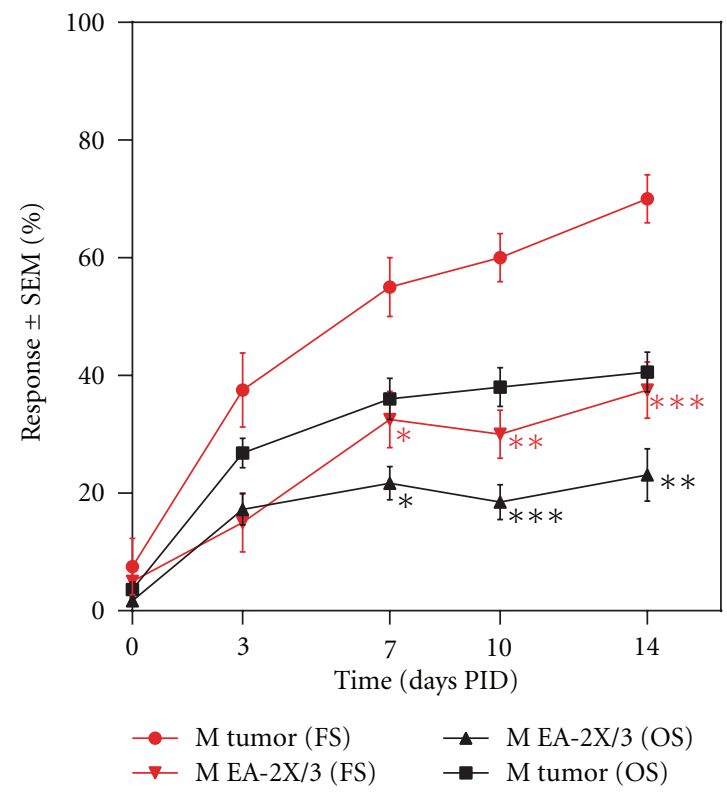

(b) M Fibrosarcoma (FS) versus M Osteosarcoma (OS)

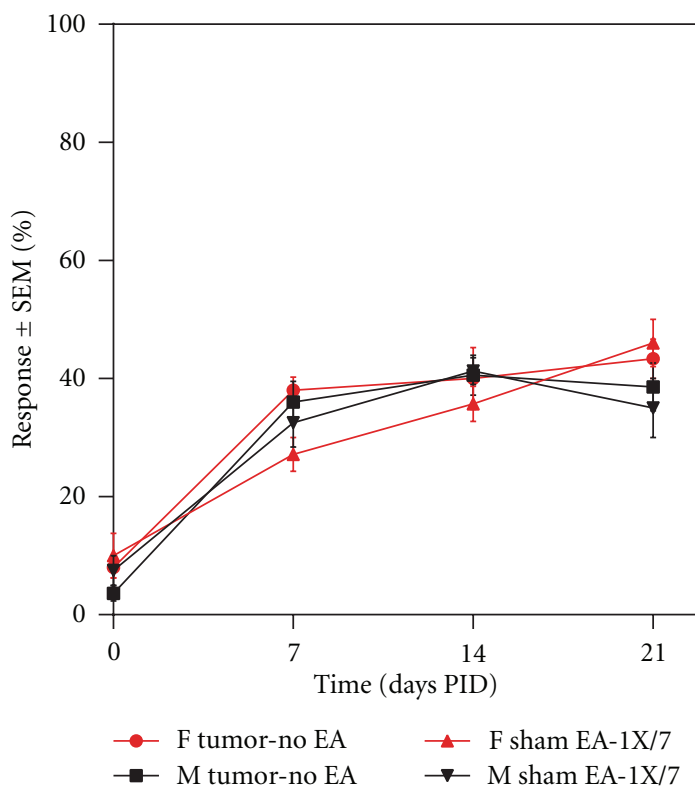

(d) MF Tumor versus MF SHAM EA-1X/7

FIGURE 1: Graphs illustrating (a) tumor-induced mechanical hyperalgesia from postimplantation day (PID) 3 to 21 in male and female mice as measured using a von Frey filament applied 10 times to the hind paw. Pronounced hyperalgesia is evident as early as PID 3 and extends through PID 21 in both male and female animals $(n=15$ /group $)$ as compared to their saline controls $\left(n=5 /\right.$ group, ${ }^{*} P<0.05$, ${ }^{* *} P<0.01, * * * P<0.001$, and $* * * * P<0.0001$ ); (b) a comparison of the effect of electroacupuncture treatment given twice per week beginning on PID 3 (EA-2X/3) on tumor-induced mechanical hyperalgesia in Bulb/c osteosarcoma male mice $(n=18 /$ group) compared to $\mathrm{C} 3 \mathrm{H}$ fibrosarcoma male mice ( $n=4$ /group). Electroacupuncture produced a significant decrease in tumor-induced hyperalgesia in both mouse strains $\left({ }^{*} P<0.05,{ }^{* *} P<0.01,{ }^{* * *} P<0.001\right)$. Note that Bulb/c mice were used for the behavioral and immunohistochemical experiments, while $\mathrm{C} 3 \mathrm{H}$ mice were used for the microperfusion experiments in this study; (c) the effect of EA administered once per week starting on PID 7 on tumor-induced hyperalgesia; and (d) the effect of sham acupuncture administered once per week starting at day 7 on tumor-induced hyperalgesia. EA-1X/7, but not sham EA, produces an anti-nociceptive effect only in female animals and only on PID 7. Data is presented as the mean \% response \pm SEM. 
once per week beginning on day 7 had no effect on tumorinduced mechanical hyperalgesia in male or female animals (Figure 1(c)).

\subsubsection{EA-2X/3 (Electroacupuncture Administered Twice per} Week Beginning PID 3). In male mice, a significant reduction in tumor-induced hyperalgesia was observed beginning at PID 7 as compared to the Tumor-No EA control group, and this reduction was evident through PID 14. In female tumor mice, a significant reduction in tumor-induced hyperalgesia was first observed at PID 10, and this continued through PID 17 (Figure 2(a); ${ }^{*} P<0.05,{ }^{* *} P<0.01$, and ${ }^{* * * *} P<$ $0.0001)$. Overall, male animals were more sensitive to EA$2 \mathrm{X}$ when compared to female counterparts, and, thus, this regimen produced an earlier and more robust antihyperalgesic effect in males. EA-2X was the only treatment regimen that produced significant analgesia at the 14 PID time point in both male and female animals, and this analgesic effect remained through PID 17 in female mice. In contrast, sham EA treatment given twice per week beginning at day 3 had no effect on tumor-induced mechanical hyperalgesia (Figure 2(b)).

3.3.3. EA-2X/5 (Electroacupuncture Administered Twice per Week Beginning PID 5). Unlike the effect of EA-2X/3 treatment, EA given twice per week beginning on PID 5 produced no long-term analgesic effect in either male or female animals (Figure 2(c)). Similarly, sham EA-2X/5 had no effect on tumor-induced mechanical hyperalgesia (Figure 2(d)).

\subsubsection{EA-ONCE/1 (Electroacupuncture Administered Once} at PID 1). While EA treatment given only once at PID 1 showed a trend towards reducing tumor-associated nociception when compared to the tumor-no EA groups, this did not reach statistical significance in either male or female animals at any of the time points tested (Figure 3(a)). Sham EA-Once/1 had no effect on tumor-induced mechanical hyperalgesia (Figure 3(b)).

3.3.5. EA-PRO (Electroacupuncture Administered 3 Times prior to Tumor Cell Implantation). Lastly, early prophylactic treatment produced a robust antihyperalgesic effect at PID 3,7 , and 10 in females and at PID 3 and 7 in male animals as compared to the tumor-no EA male and female control groups (Figure $3(\mathrm{c})$; ${ }^{* *} P<0.01,{ }^{* * *} P<0.001$, ${ }^{* * * *} P<$ $0.0001)$. This antihyperalgesic effect was absent at all of the time points tested after PID 10 (PID 14, 17, and 21). Sham EA given prophylactically (Sham EA Pro) had no effect on tumor-induced nociception.

3.3.6. EA Controls. Administration of sham EA (Sham EA1X/7, Sham EA-Once/1, Sham EA-2X/3, Sham EA-2X/5, and Sham EA Pro) had no significant effect on tumor-induced hyperalgesia at any time point tested as indicated previously (Figures 1(c), 2(b), 2(d), 3(b), and 3(d); $P>0.05$ ). Since EA$2 \mathrm{X} / 3$ produced the most robust and longest lasting analgesic effect, we repeated this regimen in a second set of animals that were used for subsequent immunohistochemical quantification of neutrophil and macrophage density within the tumor (see the following). Moreover, since this antihyperalgesic effect was evident by PID 7 in males, but not until day 10 in females, this set of experiments was only performed on male tumor and control mice. The von Frey scores for this group together with those of a sham EA$2 \mathrm{X} / 3$ and a non-acupoint treatment group (N/A-2X/3) are shown in Figure 4(a). It is noteworthy that stimulation of a nonacupoint located on the base of the tail failed to produce an analgesic effect at any of the time points tested.

\subsection{Reduction in Neutrophil but Not Macrophage Density fol-} lowing EA-2X/3 Treatment. In order to evaluate the effects of EA on tumor-associated inflammation, we utilized immunohistochemistry to quantify the density of neutrophils and macrophages present in sections through the tumor site in nontreated mice compared to those treated with EA-2X/3, sham-EA twice per week (Sham-2X/3), or non-acupoint EA twice per week (N/A-2X/3). EA-2X/3 treatment produced a significant reduction in the average neutrophil density (Figures 4(b) and 5(a)) within the tumor at PID 7 as compared to the tumor-no EA $(* * * * P<0.0001)$, Sham$2 \mathrm{X} / 3(* * P<0.01)$, and N/A-2X $(* * * P<0.001)$ groups. Image analysis measurements for average neutrophil density on PID 7 for EA-2X/3, tumor-no EA, Sham-2X/3, and N/A$2 \mathrm{X} / 3$ were $3.33 \pm 0.32,8.47 \pm 0.63,6.10 \pm 0.55$, and $6.80 \pm 0.87$, respectively (Figure 4(b)). By PID 14, neutrophil densities returned to that observed in the tumor-no EA group (Figure 4(d); $P>0.05)$. Average densities of macrophages remained comparable in all groups regardless of the time point $(P>0.05$; Figures $4(\mathrm{c}), 4(\mathrm{e})$, and $5(\mathrm{~b}))$. No nonspecific staining was observed in any of the immunohistochemical control slides.

3.5. Effect of EA-2X/3 on Tumor-Associated Prostaglandin $E_{2}$ $\left(P G E_{2}\right)$. We determined $\mathrm{PGE}_{2}$ concentrations in microperfusates taken from male $\mathrm{C} 3 \mathrm{H}$ mice both before and again following 30 minutes of EA stimulation. The data indicate that the concentration of $\mathrm{PGE}_{2}$ is increased in the extracellular fluid at the tumor site compared to saline (nontumor cell) injected control animals. Importantly, the mean tumor $\mathrm{PGE}_{2}$ concentration significantly decreased following application of EA-2X/3 (Figure 6). Finally, saline injected control animals receiving EA-2X/3 treatment also showed a reduction in $\mathrm{PGE}_{2}$ concentration compared to saline injected control animals receiving no EA treatment. Mean $\mathrm{PGE}_{2}$ concentrations for Saline, Tumor, Sal + EA-2X, and Tumor + EA-2X were $265.80 \pm 0.20,309.20 \pm 16.33$, $225.20 \pm 12.80$, and $135.20 \pm 4.36$, respectively $\left({ }^{*} P<0.05\right.$, Saline + EA-2X versus Tumor + EA-2X; ${ }^{*} P<0.05$, Tumor versus Tumor + EA-2X; ${ }^{*} P<0.05$, Saline versus Sal + EA2X).

\section{Discussion}

Acupuncture has been shown to be efficacious in the management of the side effects associated with cancer chemotherapy, and radiotherapy and more recently several 


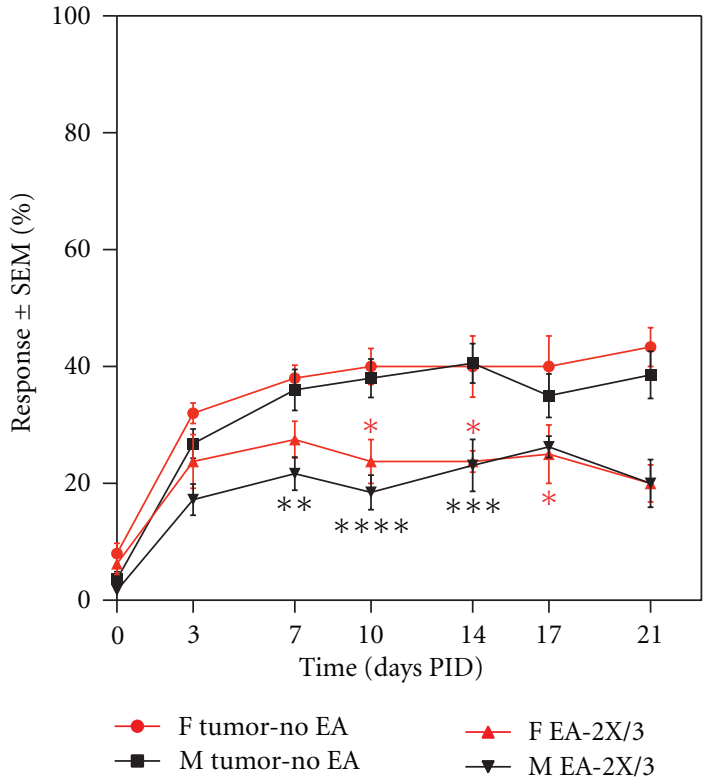

(a) MF Tumor versus MF EA-2X/3

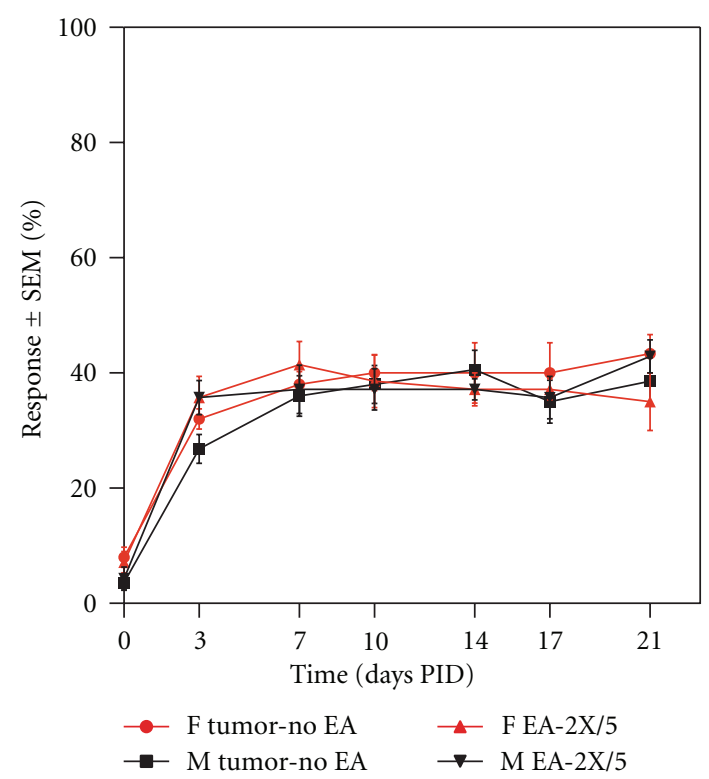

(c) MF Tumor versus MF EA-2X/5

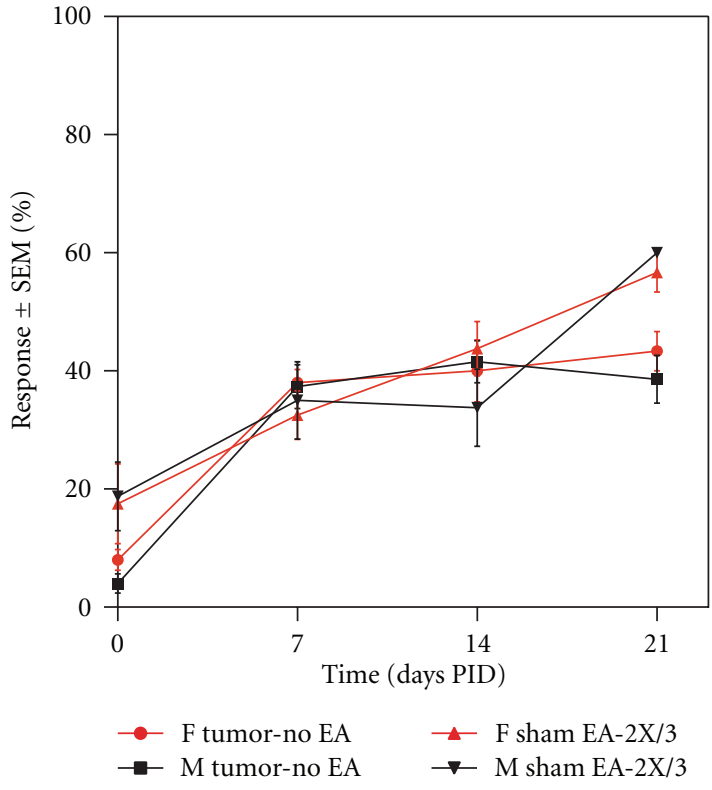

(b) MF Tumor versus MF SHAM EA-2X/3

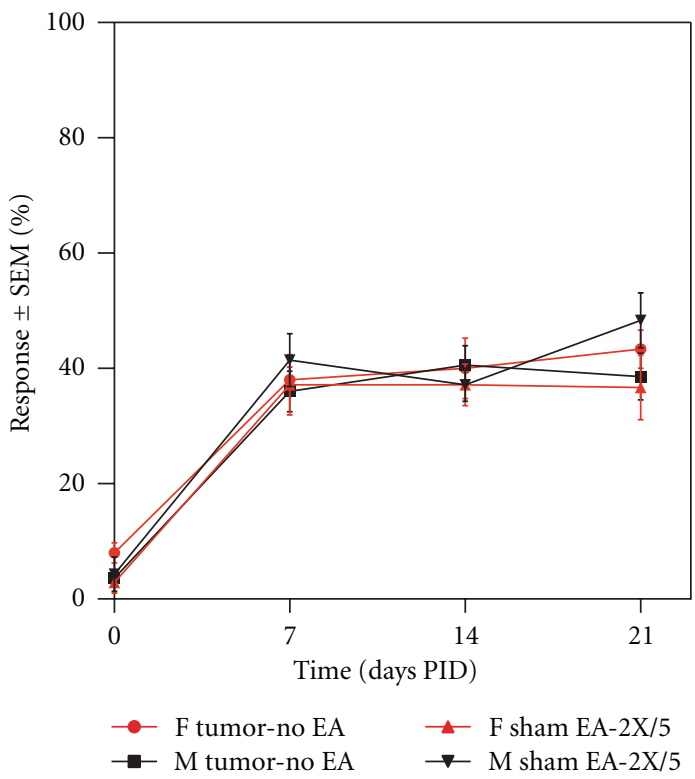

(d) MF Tumor versus MF SHAM EA-2X/5

FIGURE 2: Graphs illustrating the effect of electroacupuncture (a), (c) or sham EA (b), (d) given twice per week beginning on PID 3 (EA-2X/3) or PID 5 (EA-2X/5) on tumor-induced hyperalgesia. EA-2X/3, but not EA-2X/5 had an early and more robust effect on male (M) animals, but had a longer lasting effect on female (F) animals ( $(\mathrm{a}) ; n=12-18$ /group). EA-2X/3 produced the longest lasting anti-hyperalgesic effect of all the regimens tested. Neither sham EA-2X/3 nor sham EA-2X/5 had any effect on tumor-induced nociception. Data is presented as mean $\%$ response $\pm \mathrm{SEM},{ }^{*} P<0.05,{ }^{* *} P<0.01,{ }^{* * *} P<0.001$, and ${ }^{* * * *} P<0.0001$ as compared to the tumor-no EA control group.

studies suggest that it can also be used effectively to treat cancer pain itself $[5,7,35,46-49]$. Yet, despite the number of studies documenting a positive effect of acupuncture on tumor pain [35] two recent systematic reviews of randomized clinical trials have failed to provide strong support for a significant effect of acupuncture on cancer pain $[50,51]$. These reviews do not speak as much to the ineffectiveness of acupuncture as they do to the inadequacies and lack of rigor in the majority of acupuncture-associated randomized clinical trials in the literature, which suffer from methodological flaws. These flaws include inadequate study design, use of improper controls, poor reporting of results, lack of or inadequate blinding, small sample size, and overestimation of the results. Thus, the problem of establishing EA's effectiveness in treating cancer pain or other types of pain may not lie within the EA approach itself, 


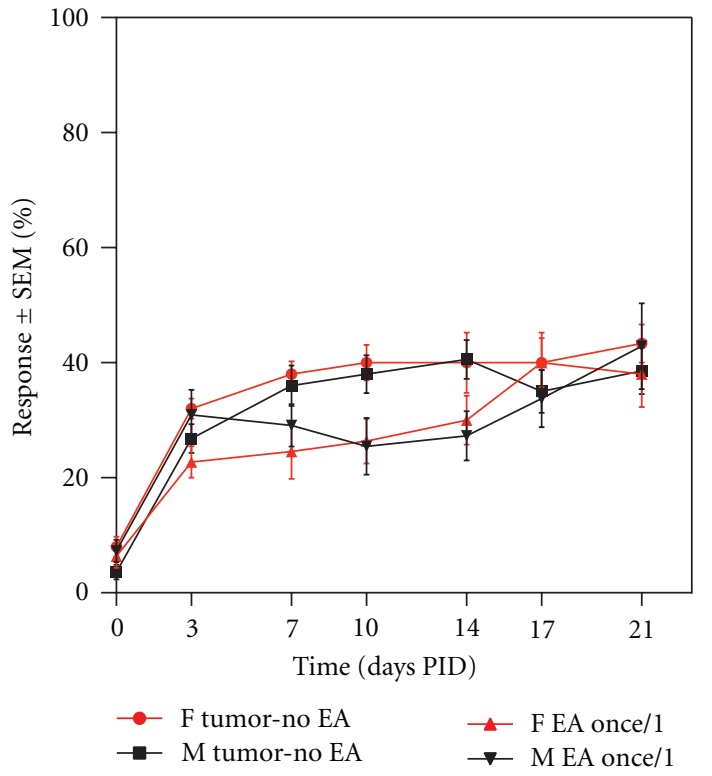

(a) MF Tumor versus MF EA ONCE/1

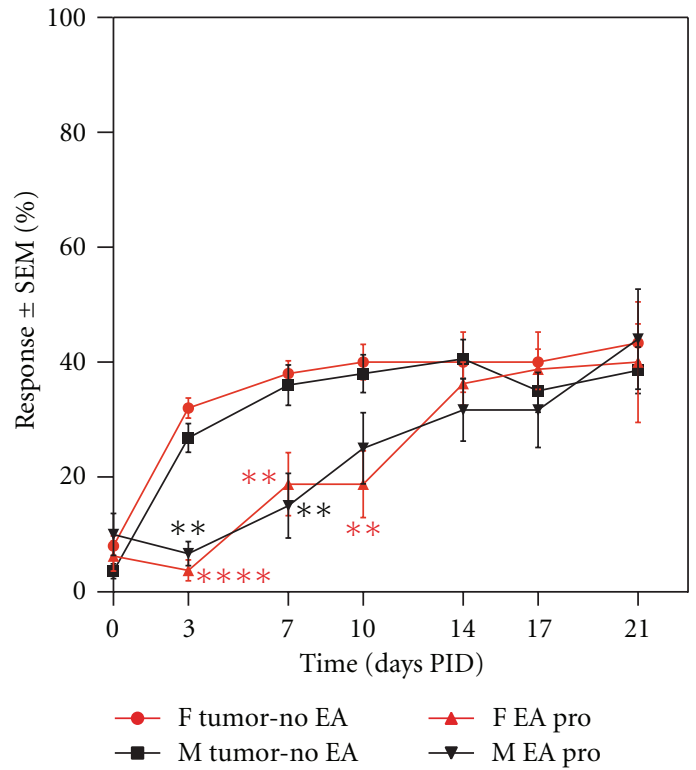

(c) MF Tumor versus MF EA PRO

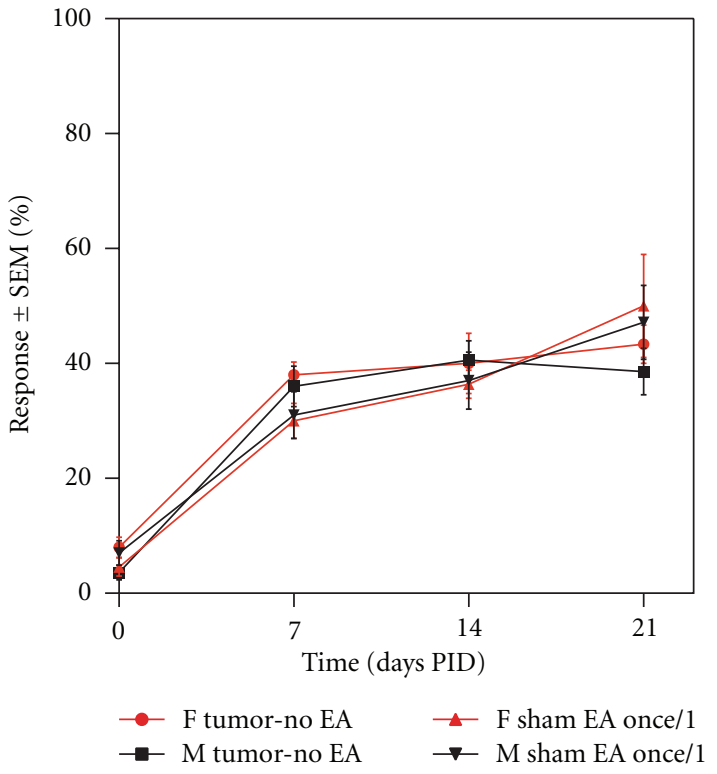

(b) MF Tumor versus MF SHAM EA ONCE/1

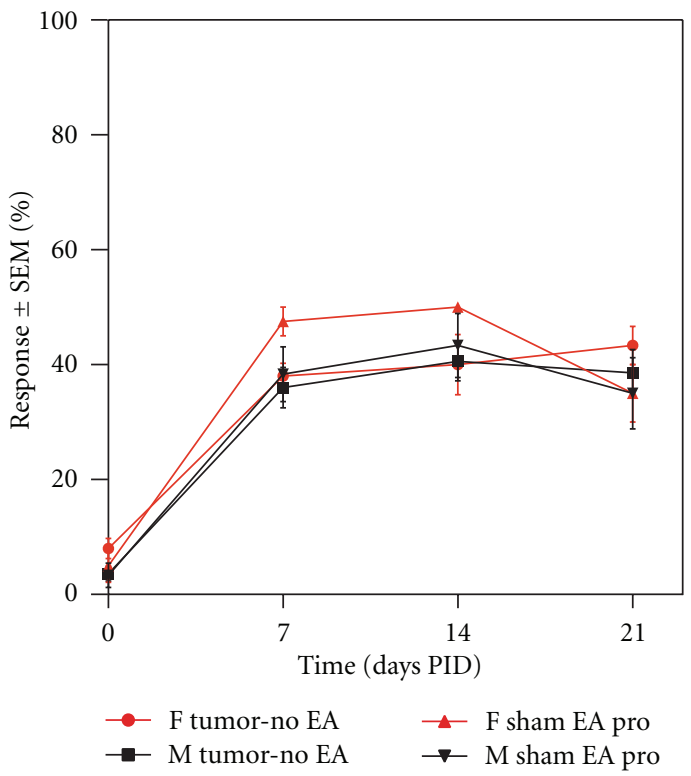

(d) MF Tumor versus MF SHAM EA PRO

FIGURE 3: Graphs illustrating the effect of EA and Sham EA administered once on PID 1 ((a) and (b), resp.) and EA or sham EA administered prophylactically prior to tumor cell implantation ((c), (d), resp.) on tumor-induced mechanical hyperalgesia. Sham electroacupuncture produced no significant effects on mechanical hyperalgesia when compared to tumor controls, $n=6-12 /$ group. Neither EA once/1 (a) nor Sham EA Once/1 (b) had any significant effect on tumor nociception. EA Pro produced an early anti-nociceptive effect beginning on PID 3 in both male and female animals (c). This effect was more robust and lasted longer in females. Sham EA Pro had no effect on tumor-induced hyperalgesia. Data shown as mean $\%$ response \pm SEM, ${ }^{* *} P<0.01$, and ${ }^{* * * *} P<0.0001$.

but rather within setting proper methodological parameters, creating the appropriate study design and sample size and establishing proper controls [5]. In this regard, there are several advantages to studying the potential antinociceptive effects of acupuncture in rodent models of cancer pain, because it is possible to control tumor development, sample size, methodological parameters, and the study design in a lab environment.
The majority of previous studies that have used animal models to analyze the potential anti-nociceptive effects of acupuncture on tumor-induced nociception provide strong support for the hypothesis that acupuncture can relieve tumor-induced pain $[6,51-54]$. Using an osteosarcoma bone cancer model, we demonstrate that different EA regimens all produce an immediate, but temporary effect lasting for approximately $30 \mathrm{~min}$ after treatment. This is consistent with 


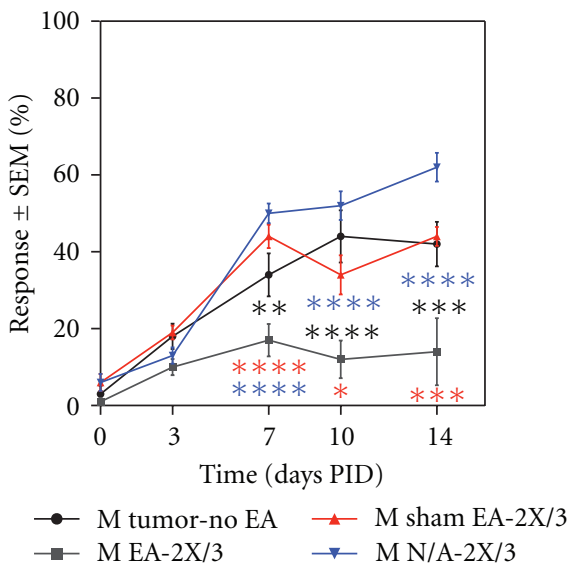

(a) Twice-Weekly EA Regimes
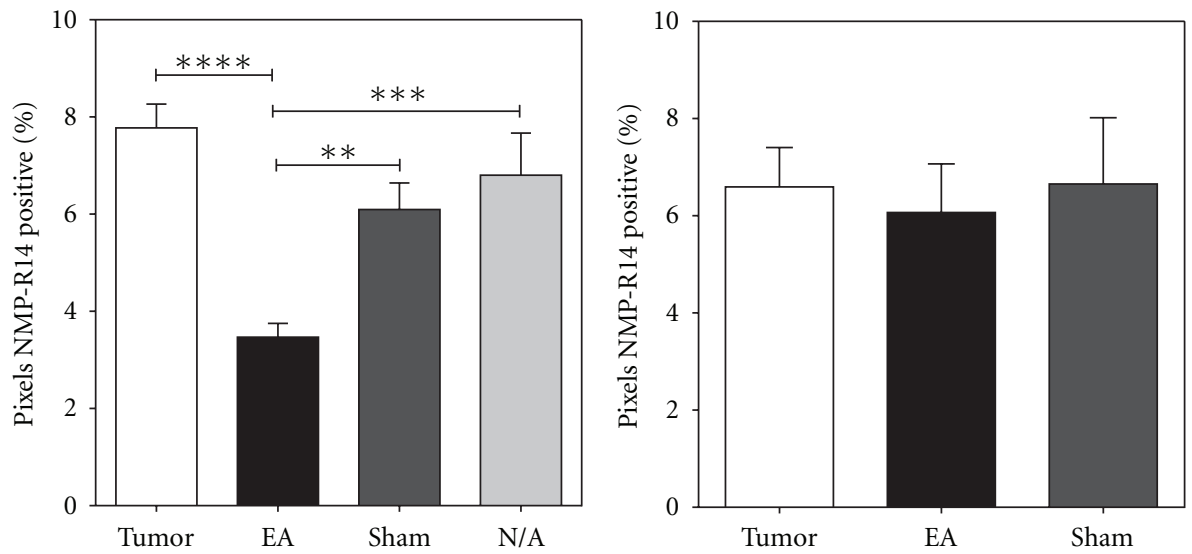

(b) NIMP-R14 immunoreactivity-Day 7 Post Implantation

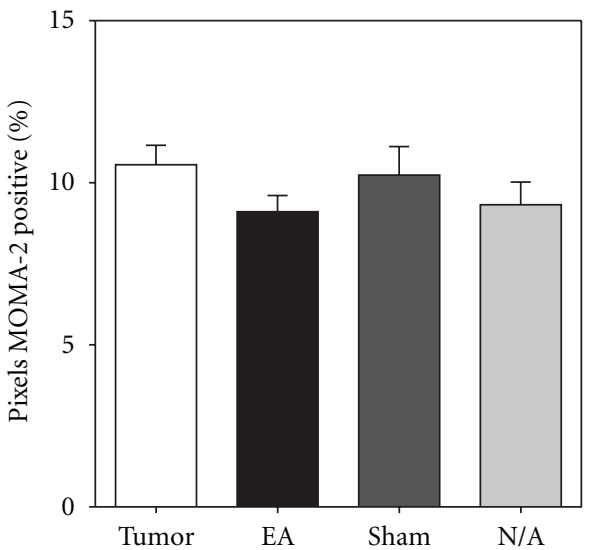

(c) NIMP-R14 immunoreactivity-Day 14 Post Implantation

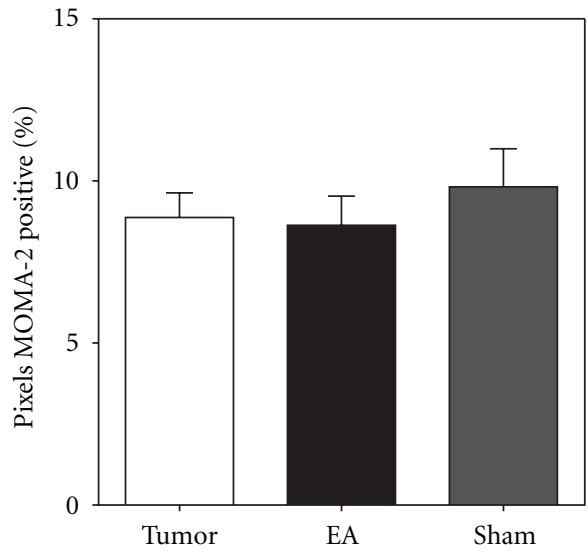

(d) MOMA-2 immunoreactivity-Day 7 Post Implantation

(e) MOMA-2 immunoreactivity-Day 14 Post Implantation

FIgURE 4: Graphs showing (a) the effect of EA-2X/3, sham EA-2X/3 and twice weekly non-acupoint electroacupuncture (N/A-2X/3) treatment on tumor-induced hyperalgesia in the mice used for this experiment and ((b) to (e)) the effects of EA-2X/3 on neutrophils and macrophages at the osteosarcoma tumor site of male (M) mice at PID 7 and 14 ( $n=5 /$ group). EA-2X/3, but neither Sham EA-2X/3 nor N/A-2X/3, significantly decreased tumor-induced hyperalgesia at PID 7, 10 and 14 ((a); $n=10-15 /$ group). Data in "(a)" shown as mean $\%$ response $\pm \mathrm{SEM} ;{ }^{*} P<0.05,{ }^{* * *} P<0.0001$ for N/A-2X. Sections were immunostained with NIMP-R14 an antibody specific for neutrophils (b) and (c) or with MOMA-2 for macrophages (d) and (e). The average percent Pixel Density (PD) of neutrophils (b), (c) and macrophages (d), (e) within the tumor were calculated from the $40 \mu \mathrm{m}$ thick immunostained sections as described in Section 2. Each bar represents the average \% PD (mean \pm SEM) of neutrophils or macrophages at the tumor site for animals treated with no EA, EA-2X, ShamEA-2X, or EA applied to a non-acupoint (M N/A-2X). Images were analyzed with ImageJ software (NIH, Bethesda, MD)—see Figure 5. EA-2X/3 treatment produced a significant reduction in the average neutrophil density within the tumor periphery at PID 7 as compared to tumor-no EA $\left({ }^{* * *} P<0.0001\right)$, Sham-2X/3 (**P $\left.<0.01\right)$, and N/A-2X/3 (*** $\left.P<0.001\right)$, returning to control densities by PID $14 ; P>0.05$ for all groups. Average densities of macrophages remained comparable in all groups regardless of the time point measured $(P>0.05)$. 


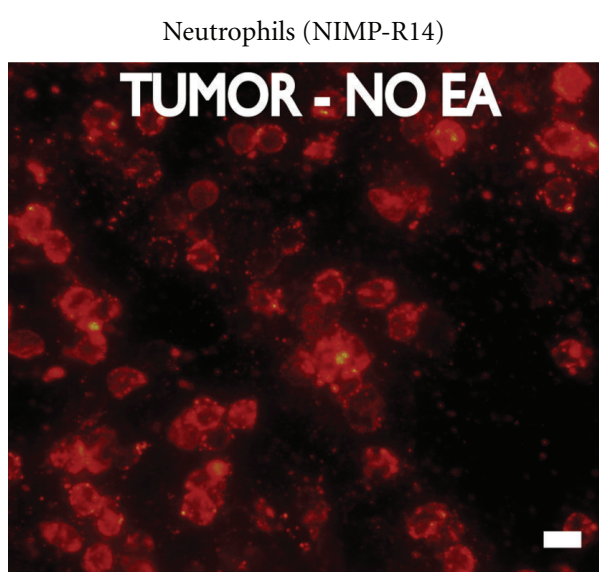

(a)

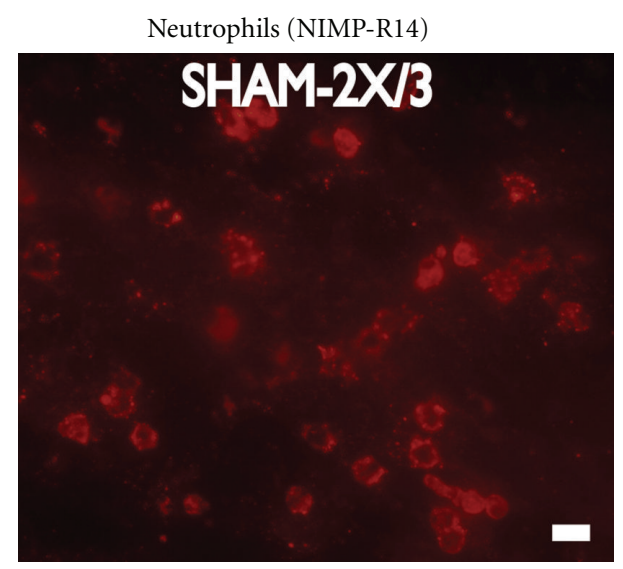

(c)

Macrophages (MOMA-2)

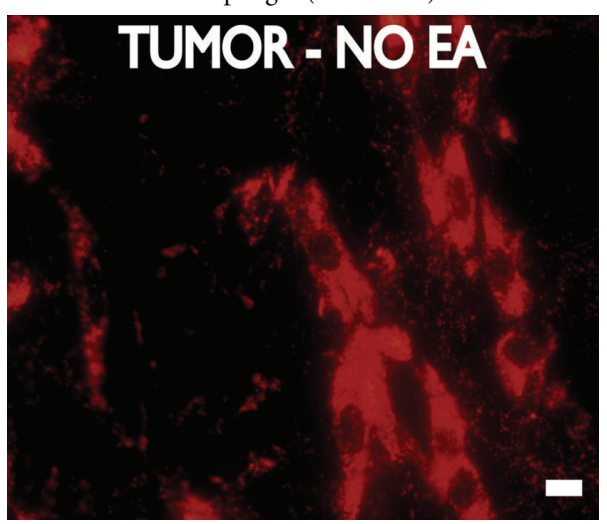

(e)

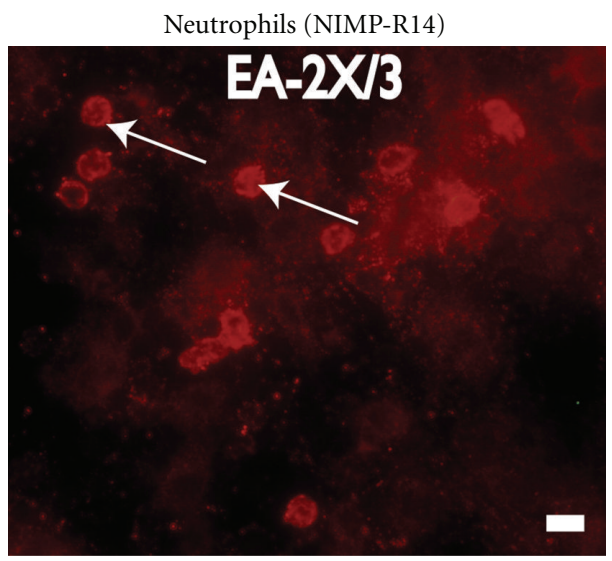

(b)

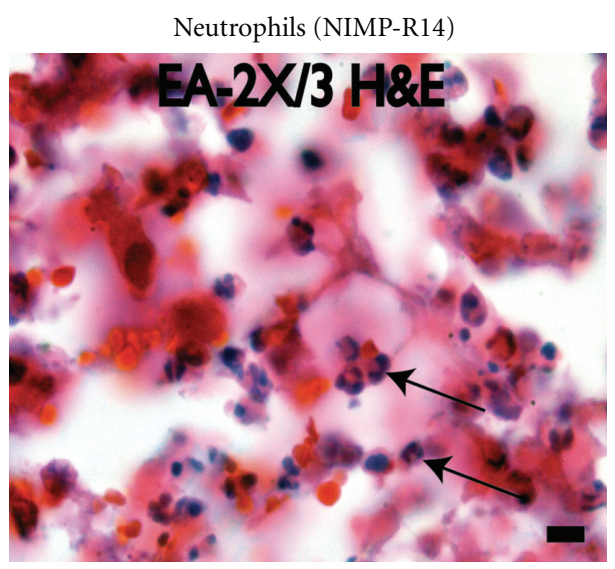

(d)

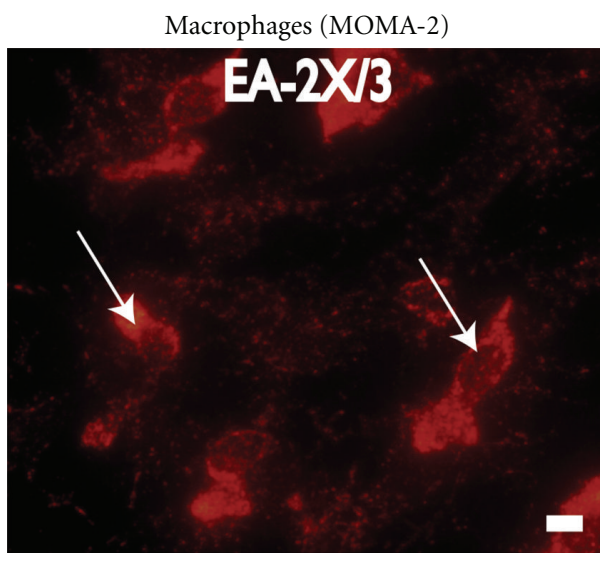

(f)

FIGURE 5: Fluorescent immunohistochemistry and H\&E staining for neutrophils (a)-(d) or macrophages (e)-(f) in peripheral tumor hind paw tissue. All images were magnified to 40X with a Nikon 80i fluorescent microscope and captured with ACT-1 software (Nikon, Melville, NY). White arrows (b), (f) indicate labeled immunocytes; black arrows (d) indicate neutrophils stained with H\&E. EA-2X/3 significantly reduced the number of neutrophils at the tumor site ((b) compared to (a)), but produced no change in the numbers of macrophages ((e) versus (f)). Scale bars represent $20 \mu \mathrm{m}$.

the findings of Mao-Ying et al. [53] who demonstrated that a single EA treatment on day 8 after implantation of melanoma cells into the hind paw produced a significant analgesic effect immediately after the treatment reaching a maximum effect within 15 to $20 \mathrm{~min}$ after EA. With respect to long-term effects, we show that only EA given twice weekly beginning at PID 3 or prophylactically prior to tumor cell implantation significantly reduced tumorinduced mechanical hyperalgesia over days to weeks. Thus, one of the novel outcomes of the present study is that 


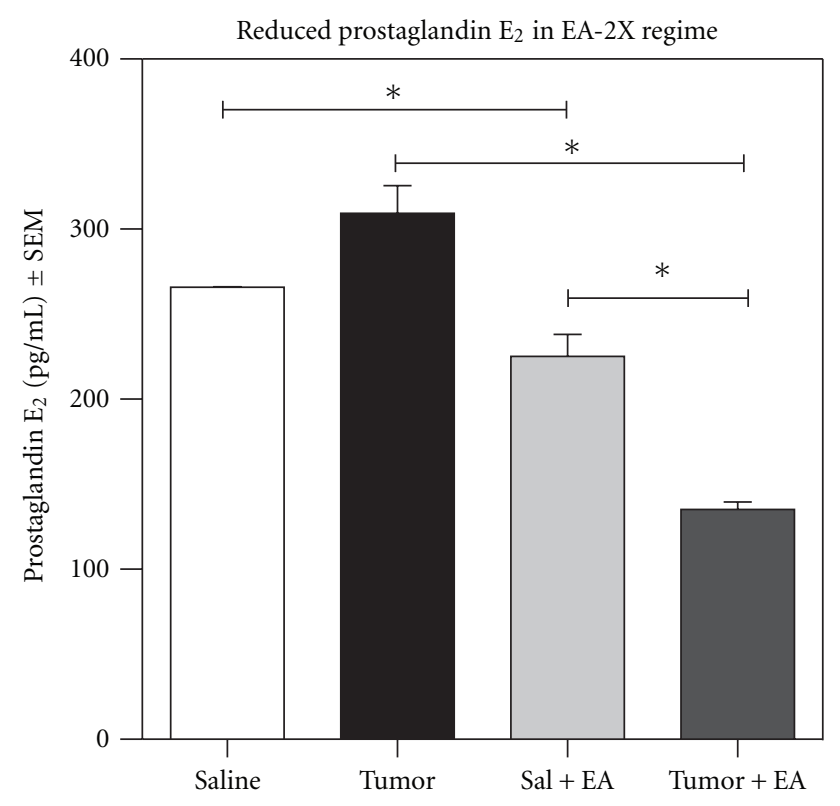

Figure 6: Mean $\mathrm{PGE}_{2}$ concentration in $\mathrm{pg} / \mathrm{mL}$ as measured by ELISA from fibrosarcoma tumor microperfusate samples $(n=$ 3 /group). Data are expressed as mean \pm SEM. Mean tumor $\mathrm{PGE}_{2}$ concentration significantly decreased following application of EA-2X beginning at PID 3. In addition, EA reduced the $\mathrm{PGE}_{2}$ concentration in saline injected control animals. Mean $\mathrm{PGE}_{2}$ concentrations for Saline, Tumor, Sal + EA-2X and Tumor + EA-2X were $265.80 \pm 0.20,309.20 \pm 16.33,225.20 \pm 12.80$, and 135.20 \pm 4.36 , respectively $\left({ }^{*} P<0.05\right.$, Saline + EA-2X versus Tumor + EA-2X; ${ }^{*} P<0.05$, Tumor versus Tumor + EA-2X; ${ }^{*} P<0.05$, Saline versus $\mathrm{Sal}+\mathrm{EA}-2 \mathrm{X})$.

different treatment regimens produce different long-term anti-nociceptive results and that early treatment given twice a week produces the most long-lasting analgesia particularly in females. Mao-Ying et al. [53] also showed that repeated EA treatments (given once every other day starting at PID 8 after melanoma implantation) showed a persistent analgesic effect, while it showed no therapeutic effect when starting from day 16. This lack of effect when EA treatment is started later is reminiscent of our finding that twice weekly treatment starting at PID 5, rather than PID 3 negates the long-term analgesic effect. The difference in timing between the two studies (treatment beginning at PID 3, but not PID 5 is effective for osteosarcoma versus PID 8, but not PID 16 for melanoma) probably reflects a combination of the different tumor models used (bone cancer versus cutaneous melanoma) and the different behavioral tests employed (von Frey versus radiant heat). In a study by Lee and coworkers [52], EA was administered to ST-36 daily for 9 days after tumor cell injection, and they reported that EA treatment significantly prolonged paw withdrawal latency from 5 days following inoculation. Similarly Zhang et al. [54] administered EA daily at GB-30 between PID 14 and 18 in a rat prostate bone cancer model and saw a significant inhibition of cancer-induced thermal hyperalgesia. While we did not attempt daily treatment in the present study, our results support the concept that early and frequent treatment (in our case EA given twice a week beginning on day 3 subsequent to tumor cell implantation) has long-lasting analgesic effects with respect to cancer pain. While we cannot make solid recommendations for the treatment of human cancer pain based on the present results in mice, our data would suggest that the earlier treatment is begun, the more effective the analgesic effect will be. Moreover, an acupuncture treatment regime that is given twice per week may be more effective than one given once per week or less often. Although we tested the effect of EA-2X/3 treatment on inflammation and $\mathrm{PGE}_{2}$ release at the tumor site, we did not test the effect of other treatment regimes on these parameters, which probably contribute to EA-2X/3's analgesic effect. Clearly more work needs to be done to determine if other treatment regimes also effect tumor-associated inflammation and/or prostaglandin concentrations.

With the exception of the work by Lund and Lundberg [55] there are no controlled studies in the literature that have actually examined the differences in the effects of acupuncture on pain in males versus females. Lund and Lundberg [55] hypothesized that gender contributes to the variable results of pain alleviation in response to acupuncture, and they showed that sensory thresholds are changed in men, but not women following acupuncture treatment. Our results show that the anti-nociceptive effect of both prophylactic EA and EA-2X/3 treatment lasts longer in female animals compared to males, but the analgesic effect of EA-2X/3 treatment begins early and is more robust in male animals. In contrast both EA-1X/7 and EA-Once/1 treatments had little effect on tumor-induced pain with only female animals showing a significant decrease at PID 7 following EA-1X/7 treatment. Collectively, this data suggests that sex differences do exist with respect to EA treatment and are consistent with a recent report showing differences in brain activity between men and women during acupuncture treatment [56]. While our data is not directly prescriptive for human acupuncture treatment regimes, it would suggest that early treatment of female cancer patients may be more beneficial, while males may benefit from more frequent treatments. However, until further study of EA sex differences in human patients is performed, it would be prudent to consider the factors of gender and sex when planning acupuncture treatment, as the response to acupuncture treatment may differ between men and women. Nonetheless, it is critical that future clinical acupuncture trials, those that are well blinded and include placebo acupuncture, take into account potential sex difference.

Over the past decade evidence has accumulated indicating that there is a strong link between inflammation and cancer [17, 22-24]. Infection-driven inflammation has been implicated in the pathogenesis of $\sim 15 \%-20 \%$ of human tumors [22], chronic inflammation appears to proceed tumorigenesis for many types of tumors [25, 57], most tumors appear to have an inflammatory component, and increasing evidence indicates that leukocyte infiltration can promote tumor phenotypes, such as angiogenesis, growth, and invasion [58]. Moreover, a wide array of proinflammatory cytokines, chemokines, prostaglandins and extracellular 
proteins are closely involved in the premalignant and malignant conversion of cells on a background of chronic inflammation [22]. While the interaction of these inflammatory mediators and effector cells with carcinogenesis and tumor progression is complicated, it is likely that the release of these mediators contributes to tumor pain [12, 59].

Acupuncture stimulation has been previously shown to reduce inflammation [60-62] and also to reduce inflammatory-induced pain [63]. The ST-36 acupoint has been used in many studies that have evaluated the antinociceptive effects of acupuncture, and this same acupoint has also been used to investigate the potential anti-inflammatory capabilities of acupuncture [61,64]. Electroacupuncture at ST-36 decreases COX-2 expression in models of pain and inflammation [65]. In the present study, we found that EA administered at ST-36 for 30 min twice per week significantly reduced the density of neutrophils at the tumor site and that this correlated with EA's anti-nociceptive effects. While we did not determine the mechanisms by which EA reduced neutrophil density in the context of the present study, previous work from our laboratories has shown that EA can reduce neutrophil migration into sites of inflammation via activation of sympathetic pathways, release of noradrenaline, and activation of beta adrenergic receptors on neutrophils to inhibit their migration $[60,66]$. Similarly, da Silva and colleagues [67] showed that stimulation of the SP-6 acupoint inhibited neutrophil infiltration in a mouse peritonitis model. While this is a likely mechanism underlying the EAinduced reduction in neutrophil densities at the tumor site, it is also possible that neutrophil density was reduced by apoptosis, since reduction through apoptotic mechanisms plays an important role in maintaining homeostasis in neutrophil numbers, which are short-lived cells and readily go into apoptosis [68].

While a reduction in neutrophil density at the tumor site may be partially responsible for the EA-associated reduction in tumor-induced nociception, this is by no means the entire story. As indicated in Figure 5(b), the density of neutrophils at the tumor site returned to that observed in tumor control animals with no EA by 14 days after tumor cell implantation, a time point at which there is still a significant EA-induced anti-nociceptive effect on tumor-induced mechanical hyperalgesia. Thus, other antinociceptive mechanisms are at play at the 14-day postimplantation time point that apparently does not involve a reduction in neutrophil density. Another potential candidate for tumor-induced nociception is prostaglandin $\mathrm{E}_{2}\left(\mathrm{PGE}_{2}\right)$. When tissue damage or inflammatory conditions are present, prostaglandins are synthesized and released in response. We have shown in the present study that the concentration of $\mathrm{PGE}_{2}$ decreases in tumor microperfusates by $30 \mathrm{~min}$ following EA-2X, and it is possible that this may be one of the factors that contribute to the reduction in tumorinduced nociception. Since $\mathrm{PGE}_{2}$ has been shown to cause pain following intraplantar injection in mice [69], it is likely that the tumor-associated increase in $\mathrm{PGE}_{2}$ contributes to tumor-induced nociception. The EA-induced reduction in extracellular $\mathrm{PGE}_{2}$ concentration at the tumor site may be partially responsible for the anti-nociceptive effect of EA.

\section{Conclusion}

This study examined the effects of five different electroacupuncture (EA) treatment schedules on murine bone tumor-induced hyperalgesia in male and female animals. We show that both early and frequent (EA-2X/3) administration of EA is effective in controlling tumor-induced hyperalgesia, while treatments applied later and less frequently are not. Importantly, we demonstrate that there are sex differences associated with different treatment regimens, which may be important to consider in treating patients clinically. In this regard the analgesic effect of both prophylactic EA and EA-2X/3 treatment lasted longer in female animals, while males showed an earlier and more robust response to EA$2 \mathrm{X} / 3$ treatment. Finally, we provide new evidence that EA may decrease tumor-induced pain in part by reducing both tumor-associated inflammation and $\mathrm{PGE}_{2}$ production at the tumor site. It is critical that EA treatment schedules be carefully considered in treating acute and chronic pain conditions particularly cancer pain and that potential sex differences be taken into account. Clearly further research needs to be done to better understand bone cancer mechanisms, how inflammation affects bone cancer progression, and if alternative medicine treatment approaches can be found that both relieve cancer pain and reduce tumor burden.

\section{Glossary}

PID: Postimplantation day

EA: Electroacupuncture

$\mathrm{PGE}_{2}$ : Prostaglandin $\mathrm{E}_{2}$

CAM: Complementary and alternative medicine.

\section{Conflict of Interests}

The authors confirm that they have no conflict of interests in relation to this work.

\section{Acknowledgments}

The authors would like to thank Elaine O'Brien and Heather Bainter for their technical assistance. This study was supported by a Grant (5R01/CA084233) from the National Cancer Institute at NIH.

\section{References}

[1] E. Ernst, "Acupuncture- a critical analysis," Journal of Internal Medicine, vol. 259, no. 2, pp. 125-137, 2006.

[2] M. I. Johnson, "The clinical effectiveness of acupuncture for pain relief-you can be certain of uncertainty," Acupuncture in Medicine, vol. 24, no. 2, pp. 71-79, 2006.

[3] M. S. Lee and E. Ernst, "Acupuncture for pain: an overview of cochrane reviews," Chinese Journal of Integrative Medicine, vol. 17, no. 3, pp. 187-189, 2011.

[4] P. Posadzki, J. Zhang, M. S. Lee, and E. Ernst, "Acupuncture for chronic nonbacterial prostatitis/chronic pelvic pain syndrome: a systematic review," Journal of Andrology, vol. 33, pp. 15-21, 2012. 
[5] C. A. Paley, M. I. Bennett, and M. I. Johnson, "Acupuncture for cancer-induced bone pain?" Evidence-based Complementary and Alternative Medicine, vol. 2011, Article ID 671043, 8 pages, 2011.

[6] Z. Zhang, C. Wang, G. Gu et al., "The effects of electroacupuncture at the ST36 (Zusanli) acupoint on cancer pain and transient receptor potential vanilloid subfamily 1 expression in walker 256 tumor-bearing rats," Anesthesia and Analgesia, vol. 114, no. 4, pp. 879-885, 2012.

[7] G. K. Donald, I. Tobin, and J. Stringer, "Evaluation of acupuncture in the management of chemotherapy-induced peripheral neuropathy," Acupuncture in Medicine, vol. 29, no. 3, pp. 230-233, 2011.

[8] R. Wong and S. Sagar, "Acupuncture treatment for chemotherapy-induced peripheral neuropathy-a case series," Acupuncture in Medicine, vol. 24, no. 2, pp. 87-91, 2006.

[9] H. S. Smith, "Painful osseous metastases," Pain Physician, vol. 14, no. 4, pp. E373-E405, 2011.

[10] H. Breivik, N. Cherny, B. Collett et al., "Cancer-related pain: a pan-European survey of prevalence, treatment, and patient attitudes," Annals of Oncology, vol. 20, no. 8, pp. 1420-1433, 2009.

[11] J. M. Jimenez Andrade and P. Mantyh, "Cancer pain: from the development of mouse models to human clinical trials," in Translational Pain Research: From Mouse To Man, L. Kruger and A. R. Light, Eds., chapter 4, CRC Press, Boca Raton, Fla, USA, 2010.

[12] T. Middlemiss, B. J. A. Laird, and M. T. Fallon, "Mechanisms of cancer-induced bone pain," Clinical Oncology, vol. 23, no. 6, pp. 387-392, 2011.

[13] C. Pacharinsak and A. Beitz, "Animal models of cancer pain," Comparative Medicine, vol. 58, pp. 220-233, 2008.

[14] T. King, A. Vardanyan, L. Majuta et al., "Morphine treatment accelerates sarcoma-induced bone pain, bone loss, and spontaneous fracture in a murine model of bone cancer," Pain, vol. 132, no. 1-2, pp. 154-168, 2007.

[15] K. J. Cheng, "Neuroanatomical basis of acupuncture treatment for some common illnesses," Acupuncture in Medicine, vol. 27, no. 2, pp. 61-64, 2009.

[16] N. M. Dhanani, T. J. Caruso, and A. J. Carinci, "Complementary and alternative medicine for pain: an evidence-based review," Current Pain and Headache Reports, vol. 15, no. 1, pp. 39-46, 2011.

[17] J. A. M. Stone and P. A. S. Johnstone, "Mechanisms of action for acupuncture in the oncology setting," Current Treatment Options in Oncology, vol. 11, no. 3-4, pp. 118-127, 2010.

[18] D. C. Choi, J. Y. Lee, Y. J. Moon, S. W. Kim, T. H. Oh, and T. Y. Yune, "Acupuncture-mediated inhibition of inflammation facilitates significant functional recovery after spinal cord injury," Neurobiology of Disease, vol. 39, no. 3, pp. 272-282, 2010.

[19] T. F. He, W. J. Yang, S. H. Zhang, C. Y. Zhang, L. B. Li, and Y. F. Chen, "Electroacupuncture inhibits inflammation reaction by upregulating vasoactive intestinal peptide in rats with adjuvant-induced arthritis," Evidence-Based Complementary and Alternative Medicine, vol. 2011, Article ID 290489, 8 pages, 2011.

[20] M. V. R. Scognamillo-Szabó, G. H. Bechara, S. H. Ferreira, and F. Q. Cunha, "Effect of various acupuncture treatment protocols upon sepsis in wistar rats," Annals of the New York Academy of Sciences, vol. 1026, pp. 251-256, 2004.
[21] S. C. Baumgarten and J. Frasor, "Minireview: inflammation: an instigator of more aggressive estrogen receptor (ER) positive breast cancers," Molecular Endocrinology, vol. 26, no. 3, pp. 360-371, 2012.

[22] J. K. Kundu and Y.-J. Surh, "Emerging avenues linking inflammation and cancer," Free Radical Biology and Medicine, vol. 52, no. 9, pp. 2013-2037, 2012.

[23] A. Maccio and C. Madeddu, "Inflammation and ovarian cancer," Cytokine, vol. 58, pp. 133-147, 2012.

[24] A. García de Vinuesa, R. Sancho, C. García-Limones et al., "Vanilloid receptor-1 regulates neurogenic inflammation in colon and protects mice from colon cancer," Cancer Research, vol. 72, no. 7, pp. 1705-1716, 2012.

[25] S. Demaria, E. Pikarsky, M. Karin et al., "Cancer and inflammation: promise for biologic therapy," Journal of Immunother$a p y$, vol. 33, no. 4, pp. 335-351, 2010.

[26] J. H. Jiang, E. J. Yang, M. G. Baek, S. H. Kim, S. M. Lee, and S. M. Choi, "Anti-inflammatory effects of electroacupuncture in the respiratory system of a symptomatic amyotrophic lateral sclerosis animal model," Neurodegenerative Diseases, vol. 8, pp. 504-514, 2011.

[27] S. L. Oke and K. J. Tracey, "The inflammatory reflex and the role of complementary and alternative medical therapies," Annals of the New York Academy of Sciences, vol. 1172, pp. 172$180,2009$.

[28] P. W. Wacnik, L. J. Eikmeier, T. R. Ruggles et al., "Functional interactions between tumor and peripheral nerve: morphology, algogen identification, and behavioral characterization of a new murine model of cancer pain," Journal of Neuroscience, vol. 21, no. 23, pp. 9355-9366, 2001.

[29] P. W. Wacnik, G. L. Wilcox, and D. R. Clohisy, "Cancer pain mechanisms and animal models of cancer pain," Pain Research \& Management, vol. 16, pp. 615-637, 2000.

[30] C. Khanna, J. Prehn, C. Yeung, J. Caylor, M. Tsokos, and L. Helman, "An orthotopic model of murine osteosarcoma with clonally related variants differing in pulmonary metastatic potential," Clinical and Experimental Metastasis, vol. 18, no. 3, pp. 261-271, 2000.

[31] J. Schmidt, G. P. Strauss, A. Schon et al., "Establishement and characterization of osteogenic cell lines from a spontaneous murine osteosarcoma," Differentiation, vol. 39, no. 3, pp. 151$160,1988$.

[32] J. A. Bell-Krotoski, E. E. Fess, J. H. Figarola, and D. Hiltz, "Threshold detection and Semmes-Weinstein monofilaments," Journal of Hand Therapy, vol. 8, no. 2, pp. 155-162, 1995.

[33] C. Huang, Z. Q. Huang, Z. P. Hu et al., "Electroacupuncture effects in a rat model of complete Freund's adjuvant-induced inflammatory pain: antinociceptive effects enhanced and tolerance development accelerated," Neurochemical Research, vol. 33, no. 10, pp. 2107-2111, 2008.

[34] S. L. Tian, X. Y. Wang, and G. H. Ding, "Repeated electroacupuncture attenuates chronic visceral hypersensitivity and spinal cord NMDA receptor phosphorylation in a rat irritable bowel syndrome model," Life Sciences, vol. 83, no. 9-10, pp. 356-363, 2008.

[35] J.-G. Lin and Y.-H. Chen, "The role of acupuncture in cancer supportive care," American Journal of Chinese Medicine, vol. 40, no. 2, pp. 219-229, 2012.

[36] K. W. Kim, H. W. Kim, J. Li, and Y. B. Kwon, "Effect of bee venom acupuncture on methamphetamine-induced 
hyperactivity, hyperthermia and Fos expression in mice," Brain Research Bulletin, vol. 84, pp. 61-68, 2011.

[37] Y. Chae, S.-I. Um, S.-H. Yi et al., "Comparison of biomechanical properties between acupuncture and non-penetrating sham needle," Complementary Therapies in Medicine, vol. 19, supplement 1, pp. S8-S12, 2011.

[38] J. H. Chiu, M. S. Chung, H. C. Cheng et al., "Different central manifestations in response to electroacupuncture at analgesic and nonanalgesic acupoints in rats: a manganese-enhanced functional magnetic resonance imaging study," Canadian Journal of Veterinary Research, vol. 67, no. 2, pp. 94-101, 2003.

[39] M. Haake, H. H. Müller, C. Schade-Brittinger et al., "German Acupuncture Trials (GERAC) for chronic low back pain: randomized, multicenter, blinded, parallel-group trial with 3 groups," Archives of Internal Medicine, vol. 167, no. 17, pp. 1892-1898, 2007.

[40] T. Lundeberg and I. Lund, "Are reviews based on sham acupuncture procedures in fibromyalgia syndrome (FMS) valid?" Acupuncture in Medicine, vol. 25, no. 3, pp. 100-106, 2007.

[41] C. Gunn, "Motor points and motor lines," American Journal of Acupuncture, vol. 5, pp. 55-58, 1978.

[42] E. Haker and T. Lundeberg, "Acupuncture treatment in epicondylalgia: a comparative study of two acupuncture techniques," Clinical Journal of Pain, vol. 6, no. 3, pp. 221-226, 1990.

[43] K. K. S. Hui, J. Liu, O. Marina et al., "The integrated response of the human cerebro-cerebellar and limbic systems to acupuncture stimulation at ST 36 as evidenced by fMRI," NeuroImage, vol. 27, no. 3, pp. 479-496, 2005.

[44] H. M. Langevin, D. L. Churchill, and M. J. Cipolla, "Mechanical signaling through connective tissue: a mechanism for the therapeutic effect of acupuncture," The FASEB Journal, vol. 15, no. 12, pp. 2275-2282, 2001.

[45] J. P. Väyrynen, J. O. Vornanen, S. Sajanti, J. P. Böhm, A. Tuomisto, and M. J. Mäkinen, "An improved image analysis method for cell counting lends credibility to the prognostic significance of T cells in colorectal cancer," Virchows Archiv, vol. 460, no. 5, pp. 455-465, 2012.

[46] D. Walsh, "Advances in opioid therapy and formulations," Supportive Care in Cancer, vol. 13, no. 3, pp. 138-144, 2005.

[47] B. R. Cassileth, G. E. Deng, J. E. Gomez, P. A. S. Johnstone, N. Kumar, and A. J. Vickers, "Complementary therapies and integrative oncology in lung cancer: ACCP evidence-based clinical practice guidelines (2nd edition)," Chest, vol. 132, supplement 3, pp. 340S-354S, 2007.

[48] W. Lu, E. Dean-Clower, A. Doherty-Gilman, and D. S. Rosenthal, "The value of acupuncture in cancer care," Hematology/Oncology Clinics of North America, vol. 22, no. 4, pp. 631-648, 2008.

[49] O. Minton and I. J. Higginson, "Electroacupuncture as an adjunctive treatment to control neuropathic pain in patients with cancer," Journal of Pain and Symptom Management, vol. 33, no. 2, pp. 115-117, 2007.

[50] T. Y. Choi, M. S. Lee, T. H. Kim, C. Zaslawsski, and E. Ernst, "Acupuncture for the treatment of cancer pain: a systemic review of randomised clinical trials," Supportive Care in Cancer, vol. 20, no. 6, pp. 1147-1158, 2012.

[51] A. S. Hopkins Hollis, "Acupuncture as a treatment modality for the management of cancer pain: the state of the science," Oncology nursing forum, vol. 37, no. 5, pp. E344-E348, 2010.

[52] H. J. Lee, J. H. Lee, E. O. Lee et al., "Substance P and beta endorphin mediate electroacupuncture induced analgesic activity in mouse cancer pain model," Acupuncture \& ElectroTherapeutics Research, vol. 34, no. 1-2, pp. 27-40, 2009.

[53] Q. L. Mao-Ying, K. M. Cui, Q. Liu et al., "Stage-dependent analgesia of electro-acupuncture in a mouse model of cutaneous cancer pain," European Journal of Pain, vol. 10, no. 8, pp. 689-694, 2006.

[54] R. X. Zhang, A. Li, B. Liu et al., "Electroacupuncture attenuates bone cancer pain and inhibits spinal interleukin- $1 \beta$ expression in a rat model," Anesthesia and Analgesia, vol. 105, no. 5, pp. 1482-1488, 2007.

[55] I. Lund and T. Lundeberg, "On the threshold-evaluation of variability in effects of acupuncture in a gender perspective," Chinese Medicine, vol. 5, article 32, 2010.

[56] W. Q. Qiu, J. Claunch, J. Kong et al., "The effects of acupuncture on the brain networks for emotion and cognition: an observation of gender differences," Brain Research, vol. 1362, pp. 56-67, 2010.

[57] L. Zitvogel, O. Kepp, L. Galluzzi, and G. Kroemer, "Inflammasomes in carcinogenesis and anticancer immune responses," Nature Immunology, vol. 13, no. 4, pp. 343-351, 2012.

[58] N. Eiró and F. J. Vizoso, "Inflammation and cancer," World Journal of Gastrointestinal Surgery, vol. 4, pp. 62-72, 2012.

[59] B. J. A. Laird, A. C. Scott, L. A. Colvin et al., "Cancer pain and its relationship to systemic inflammation: an exploratory study," Pain, vol. 152, no. 2, pp. 460-463, 2011.

[60] H. W. Kim, D. K. Uh, S. Y. Yoon et al., "Low-frequency electroacupuncture suppresses carrageenan-induced paw inflammation in mice via sympathetic post-ganglionic neurons, while high-frequency EA suppression is mediated by the sympathoadrenal medullary axis," Brain Research Bulletin, vol. 75, no. 5, pp. 698-705, 2008.

[61] Y. B. Kwon, J. D. Lee, H. J. Lee et al., "Bee venom injection into an acupuncture point reduces arthritis associated edema and nociceptive responses," Pain, vol. 90, no. 3, pp. 271-280, 2001.

[62] E. J. Yang, J. H. Jiang, S. M. Lee, H. S. Hwang, M. S. Lee, and S. M. Choi, "Electroacupuncture reduces neuroinflammatory responses in symptomatic amyotrophic lateral sclerosis model," Journal of Neuroimmunology, vol. 223, no. 1-2, pp. 8491, 2010.

[63] L. Lao, G. Zhang, F. Wei, B. M. Berman, and K. Ren, "Electroacupuncture attenuates behavioral hyperalgesia and selectively reduces spinal Fos protein expression in rats with persistent inflammation," Journal of Pain, vol. 2, no. 2, pp. 111-117, 2001.

[64] P. D. Moon, H. J. Jeong, S. J. Kim et al., "Use of electroacupuncture at ST36 to inhibit anaphylactic and inflammatory reaction in mice," NeuroImmunoModulation, vol. 14, no. 1, pp. 24-31, 2007.

[65] J. H. Lee, K. J. Jang, Y. T. Lee, Y. H. Choi, and B. T. Choi, "Electroacupuncture inhibits inflammatory edema and hyperalgesia through regulation of cyclooxygenase synthesis in both peripheral and central nociceptive sites," American Journal of Chinese Medicine, vol. 34, no. 6, pp. 981-988, 2006.

[66] H. W. Kim, S. Y. Kang, S. Y. Yoon et al., "Low-frequency electroacupuncture suppresses zymosan-induced peripheral inflammation via activation of sympathetic post-ganglionic neurons," Brain Research, vol. 1148, no. 1, pp. 69-75, 2007.

[67] M. D. da Silva, G. Guginski, M. F. Werner, C. H. Baggio, R. Marcon, and A. R. Santos, "Involvement of interleukin-10 in the anti-inflammatory effect of Sanyinjiao (SP6) acupuncture in a mouse model of peritonitis," Evidence-based Complementary and Alternative Medicine, vol. 2011, Article ID 217946, 9 pages, 2011. 
[68] J. G. Filep and D. El Kebir, "Neutrophil apoptosis: a target for enhancing the resolution of inflammation," Journal of Cellular Biochemistry, vol. 108, no. 5, pp. 1039-1046, 2009.

[69] C. A. L. Kassuya, J. Ferreira, R. F. Claudino, and J. B. Calixto, "Intraplantar PGE2 causes nociceptive behaviour and mechanical allodynia: the role of prostanoid e receptors and protein kinases," British Journal of Pharmacology, vol. 150, no. 6, pp. 727-737, 2007. 


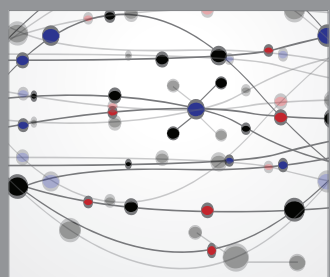

The Scientific World Journal
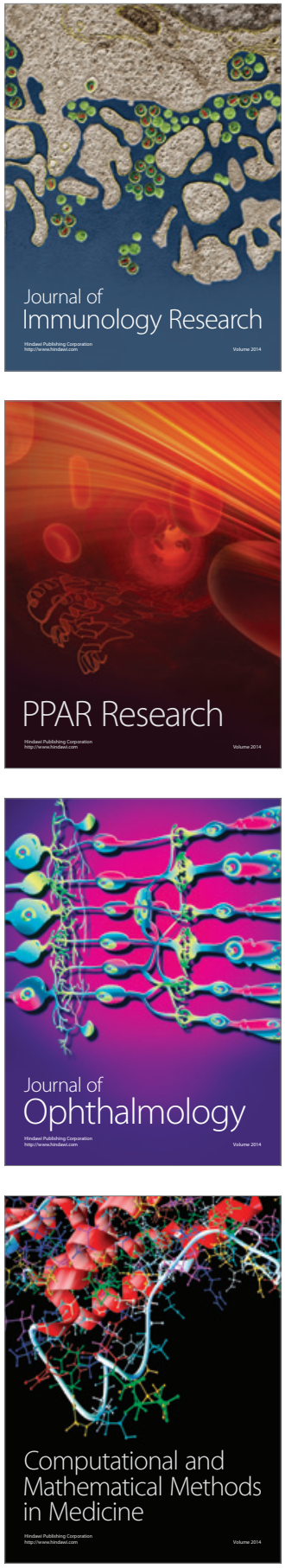

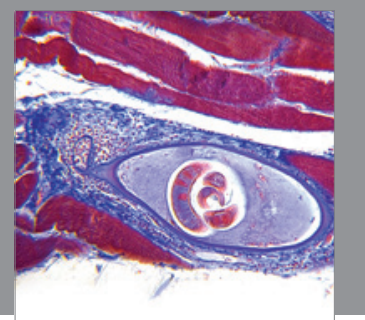

Gastroenterology

Research and Practice
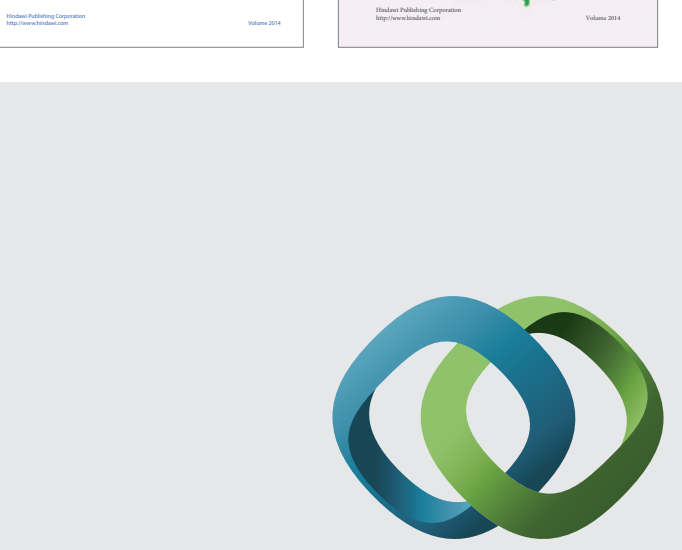

\section{Hindawi}

Submit your manuscripts at

http://www.hindawi.com
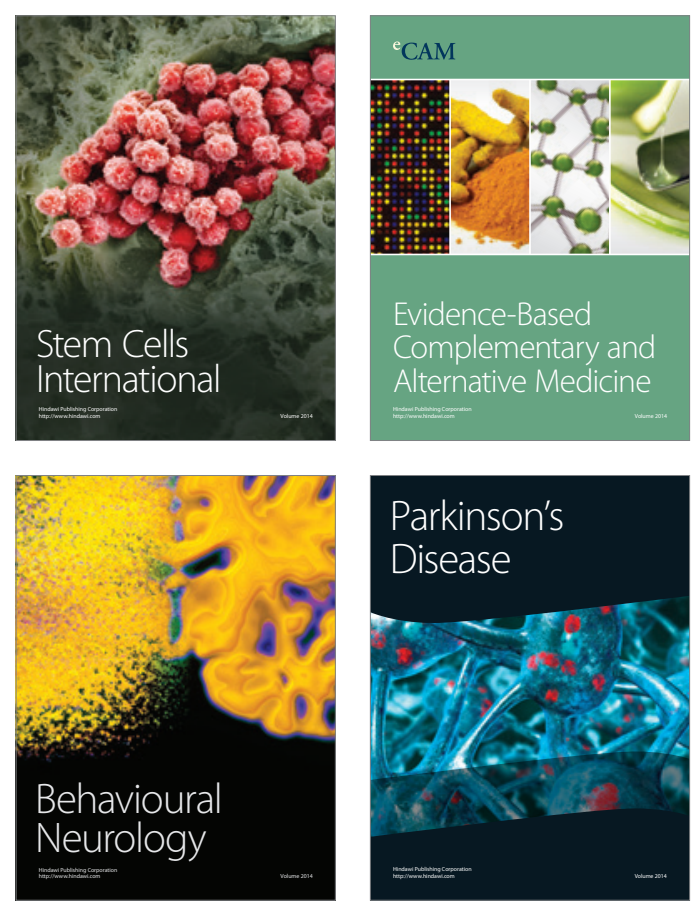

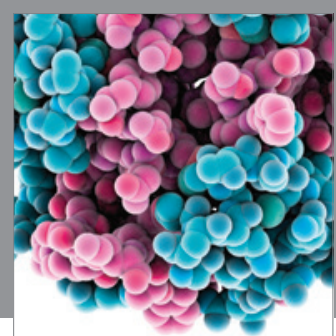

Journal of
Diabetes Research

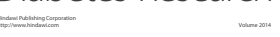

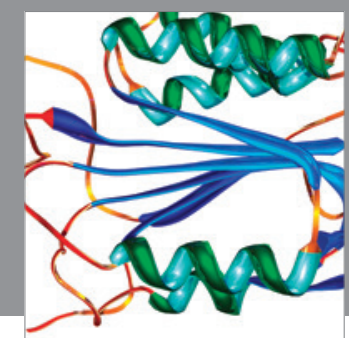

Disease Markers
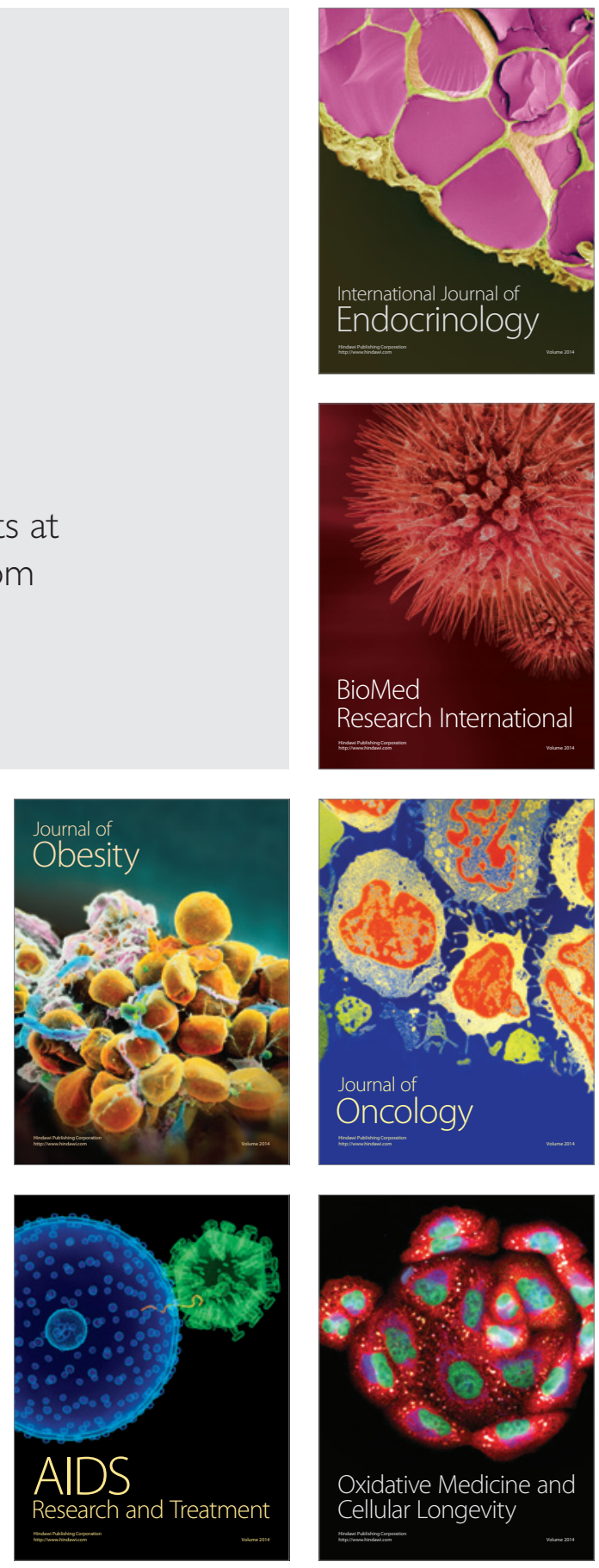